\title{
SoxNeuro orchestrates central nervous system specification and differentiation in Drosophila and is only partially redundant with Dichaete
}

\author{
Enrico Ferrero ${ }^{1,2}$, Bettina Fischer ${ }^{1,2}$ and Steven Russell ${ }^{1,2^{*}}$
}

\begin{abstract}
Background: Sox proteins encompass an evolutionarily conserved family of transcription factors with critical roles in animal development and stem cell biology. In common with vertebrates, the Drosophila group B proteins SoxNeuro and Dichaete are involved in central nervous system development, where they play both similar and unique roles in gene regulation. Sox genes show extensive functional redundancy across metazoans, but the molecular basis underpinning functional compensation mechanisms at the genomic level are currently unknown.

Results: Using a combination of genome-wide binding analysis and gene expression profiling, we show that SoxNeuro directs embryonic neural development from the early specification of neuroblasts through to the terminal differentiation of neurons and glia. To address the issue of functional redundancy and compensation at a genomic level, we compare SoxNeuro and Dichaete binding, identifying common and independent binding events in wild-type conditions, as well as instances of compensation and loss of binding in mutant backgrounds.

Conclusions: We find that early aspects of group B Sox functions in the central nervous system, such as stem cell maintenance and dorsoventral patterning, are highly conserved. However, in contrast to vertebrates, we find that Drosophila group B1 proteins also play prominent roles during later aspects of neural morphogenesis. Our analysis of the functional relationship between SoxNeuro and Dichaete uncovers evidence for redundant and independent functions for each protein, along with unexpected examples of compensation and interdependency, thus providing new insights into the general issue of transcription factor functional redundancy.
\end{abstract}

\section{Background}

The evolution of multicellular organisms is, to a large extent, driven by an increase in the complexity of gene regulatory networks [1], both at the level of cis-regulatory elements [2] and of transcription factor (TF) diversity [3]. In metazoans, many TFs have arisen through local tandem or whole genome duplications followed by neofunctionalisation, a process leading to the generation of new regulatory networks or the modification of existing ones. These processes generate developmental diversity and ultimately species evolution. Interestingly, some duplicated genes can maintain redundant functions over very substantial periods of time [4], an observation that

\footnotetext{
* Correspondence: sr120@cam.ac.uk

'Department of Genetics, University of Cambridge, Downing Street, CB2 3EH Cambridge, UK

${ }^{2}$ Cambridge Systems Biology Centre, University of Cambridge, Tennis Court

Road, CB2 1QR Cambridge, UK
}

appears to be counterintuitive from the perspective of natural selection. In general, it is expected that duplicated genes either diverge to generate new functions or one of the paralogs is lost through the accumulation of inactivating mutations [5]. It has been suggested that redundancy may be maintained when duplicates have multiple functions, both common and unique, that would otherwise be eliminated by deleterious mutations [4]. While such models account for the maintenance of closely related coding sequences in the genome, they do not explain why redundant copies do not always diverge to adopt different expression domains [5]. In some cases, it is possible that maintaining partially redundant genes with similar expression patterns may contribute to network robustness [6]; however, we lack sufficient data on the genome-wide activities of paralogous TFs to make reliable inferences about the molecular mechanisms underlying redundancy. 
The Hox family of TFs, which share a conserved organisation and function during embryonic segmentation, exemplifies the expected evolutionary trajectory of duplication events [7], with paralogous genes showing divergent expression domains and strong phenotypes when individually deleted, although analysis of double mutants suggests a limited degree of functional redundancy in some cases [8]. In contrast, Sox (SRY-related high-mobility-group box) genes, another family of metazoan TFs that have arisen through gene duplications [9-11], exhibit a much higher degree of functional redundancy, with closely related genes often widely coexpressed and able to substantially compensate for each other's loss [12-17]. The reasons why some TF families have functionally diverged while others have maintained considerable redundancy is a fascinating unanswered question.

Sox proteins have established roles in transcriptional regulation and may also play an architectural role in chromatin organisation $[18,19]$. The 20 Sox genes in vertebrates are subdivided into 8 groups (A to $\mathrm{H}$ ), most of which contain multiple paralogs. Group B genes are of particular interest from an evolutionary perspective, providing examples of both neofunctionalisation and redundancy. This group is divided into two further subgroups, B1 (Sox1, Sox2, and Sox3) and B2 (Sox14 and Sox21) [10], both playing important roles during vertebrate neurogenesis. SoxB1 proteins primarily act as transcriptional activators, in particular regulating the maintenance of neural stem cell (NSC) self-renewal, while SoxB2 proteins mainly function as transcriptional repressors, promoting the differentiation of neural precursors into mature neurons [20-22]. In most vertebrates, the three B1 proteins are extensively coexpressed in the developing central nervous system (CNS) and single gene mutants or knockdowns show only mild embryonic CNS phenotypes [23-25]. In zebrafish, where four group B1 genes are coexpressed in the CNS, only knockdown of all four elicits a severe CNS phenotype, with single, double and even triple mutant combinations showing substantial CNS development [26]. On the one hand, the evolution of diversified roles for B1 and B2 proteins illustrates neofunctionalisation, but on the other, the extensive coexpression of B1 proteins in the early CNS across the vertebrates represents a prime example of conserved functional redundancy.

The Drosophila melanogaster genome encodes four group B genes (SoxNeuro (SoxN), Dichaete (D), Sox21a, Sox21b) [27]. While there is still some uncertainty regarding the B1 and B2 subdivision in insects, with different views on their grouping and evolution proposed [10,11,28,29], at a functional level SoxB factors appear to be functionally conserved across the metazoa, with mammalian SoxB1 proteins able to rescue Drosophila mutations [30,31]. While the functions of Sox21a and Sox $21 b$ are currently unknown [27], SoxN and Dichaete have prominent roles in CNS development and exhibit extensive functional redundancy [32,33]. Both genes are dynamically expressed in partially overlapping domains of the embryonic CNS [34-38] and double mutants display far more severe CNS phenotypes than either single mutant. Along with redundant functions, each gene has unique expression domains and, in some circumstances, the two TFs also appear to have opposite functions in gene regulation $[32,33,38,39]$. The conservation in group B Sox function, combined with the evidence that individual members can have both unique and redundant functions, makes Drosophila an attractive system for studying redundancy between paralogous TFs.

SoxN and Dichaete are involved in many of the pathways controlling neural specification in Drosophila and there are striking similarities to the roles played by vertebrate group B Sox proteins that suggest an underlying conservation [20]. For example, vertebrate B1 proteins have critical roles in the maintenance of NSCs [40,41] and both fly genes are required for the correct establishment of neuroblasts (NBs), the fly equivalent of vertebrate NSCs [33,39], with Dichaete known to be involved in maintaining embryonic and larval NBs in a self-renewing state [42]. At the molecular level, Dichaete interacts with the POU protein Ventral veins lacking $(\mathrm{Vvl})$ to regulate gene expression in the CNS midline [43], a role reminiscent of the Sox2-Oct4 interaction required for stem cell maintenance in mammals [44-46]. Similarly, a set of homeodomain proteins are critical for patterning the dorsoventral (DV) axis of the CNS in both vertebrates and Drosophila, where they are coexpressed and interact with SoxB proteins $[20,33,38,47]$. Despite these known functional and molecular similarities, how widely group B Sox functions are conserved between invertebrates and vertebrates remains to be determined.

Genome-wide studies analysing global gene expression changes or patterns of genomic binding can provide significant insights into the function of TFs. Recently, a genome-wide study in mouse neural cells highlighted extensive overlap between Sox 2 and Sox3 binding, supporting the view that SoxB1 proteins are functionally redundant [48]. In Drosophila, genome-wide analysis of Dichaete implicates it in the regulation of hundreds of genes in the CNS $[39,49]$. Here, we focus on a genomic analysis of SoxN, identifying hundreds of putative direct SoxN target genes. We then tackle the issues of redundancy and compensation between SoxN and Dichaete by generating binding profiles for both factors in wild-type and mutant backgrounds. We identify instances of redundancy and compensation at the molecular level, as well as other changes in the binding profiles indicative of interdependency between the two factors. Our comparative analysis provides the first molecular view of functional redundancy and compensation between paralogous TFs at a genome- 
wide scale, and provides new insights into the functional conservation of group B Sox genes in animals.

\section{Results}

\section{Gene expression changes in SoxN mutants}

We recently performed genomic analyses of the role of Dichaete in the embryonic nervous system that identified hundreds of target genes with diverse roles in CNS development $[39,49]$. Here, we determine the functions of SoxN during embryonic development by profiling temporal changes in the transcriptome of SoxN mutants and by mapping the genome-wide binding of SoxN. To capture expression changes and binding events relevant to neural development, from the specification of NBs through to the terminal differentiation of neurons and glial cells, experiments were performed at specific time points encompassing stages 7 to 13 of embryogenesis (Figure 1A).

We extracted RNA from SoxN hemizygous null mutants and compared it with RNA from their heterozygous siblings via biologically replicated hybridisations to long oligonucleotide microarrays, across five developmental time points. After normalisation and statistical thresholding of these data, a total of 1,783 probes, corresponding to 1,665 genes, were differentially expressed across the time course (Table S1A-C in Additional file 1). At each time point, a score of $-1,0$ or 1 was attributed to all genes showing a significant differential expression according to the corrected $P$-value associated with a moderated F-statistic and the direction of the expression change

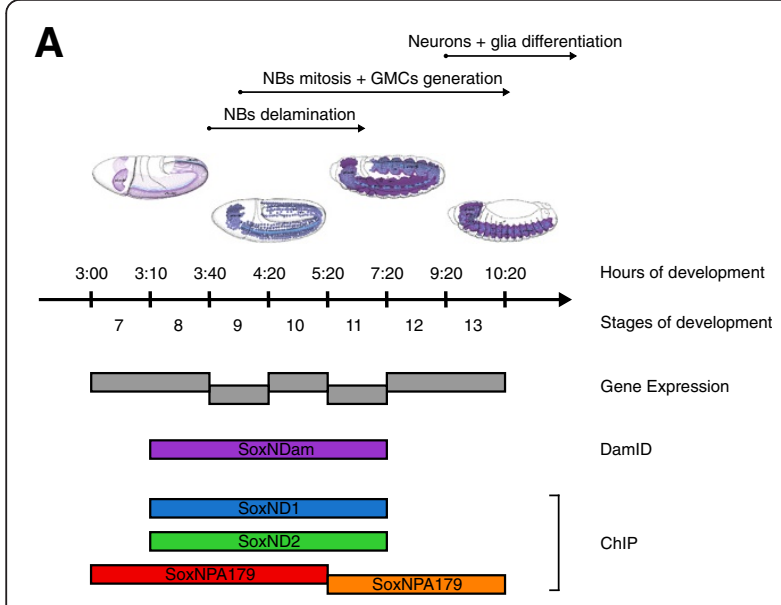

B
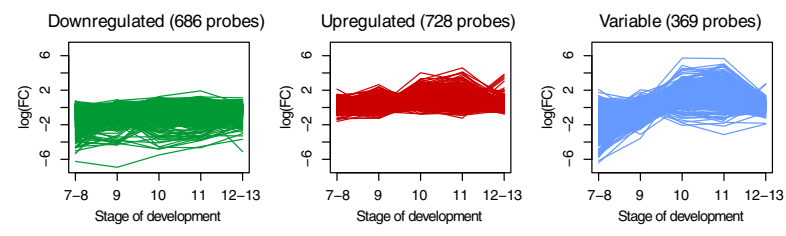
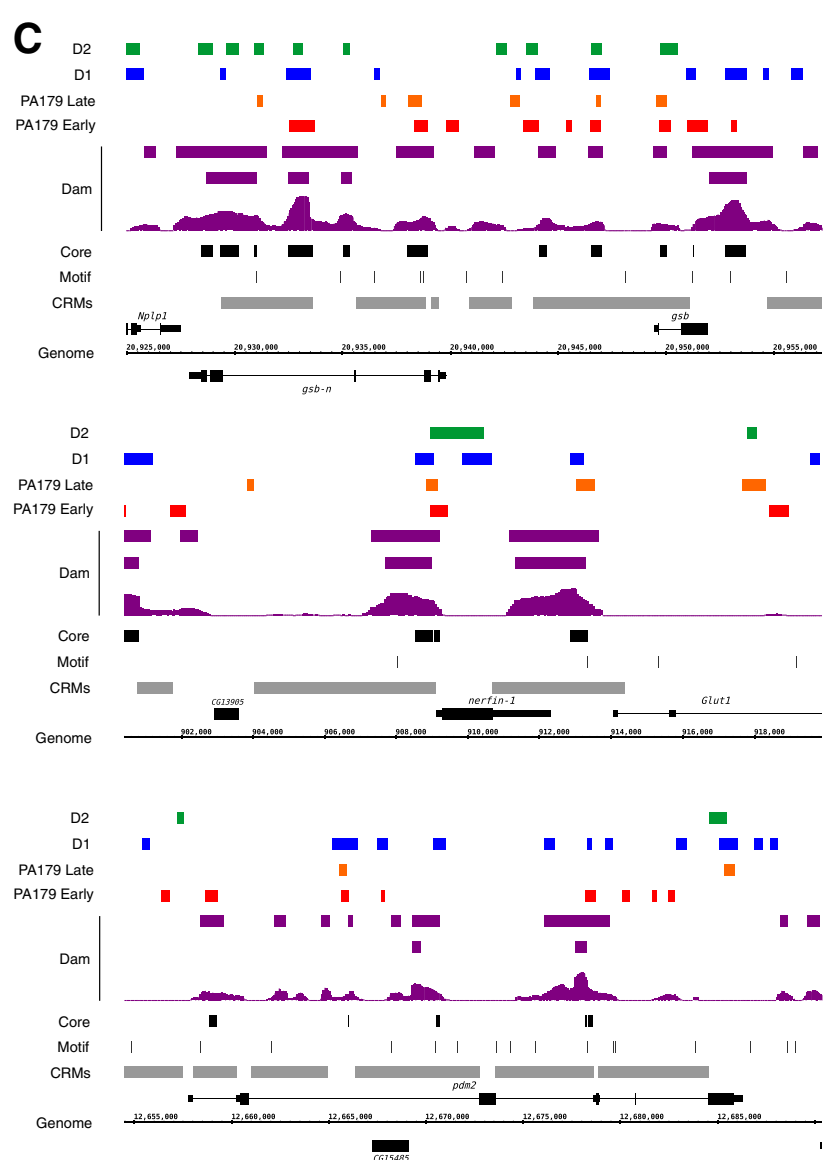

Figure 1 SoxN functional studies. (A) Overview of the SoxN datasets generated. Each coloured box below the time line represents a single gene expression, DNA adenine methyltransferase identification (DamID) or chromatin immunoprecipitation (ChIP) experiment performed at the indicated time ranges. Above the time line, major events in neural development are indicated and illustrated with FlyBase images adapted from Volker Hartenstein, Atlas of Drosophila Development, Cold Spring Harbor Laboratory Press, 1993. (B) Partitioning of genes differentially expressed in SoxN mutants. Probes corresponding to differentially expressed genes were divided into three groups (downregulated, upregulated and variable) according to their expression trend over time. (C) Genomic profiles of SoxN binding. Window scores and binding intervals at a false discovery rate (FDR) of $1 \%$ and FDR of $25 \%$ are displayed for the SoxNDam dataset (purple), and binding intervals at FDR $25 \%$ are shown for the SoxNPA179 Early (red), SoxPA179 Late (orange), SoxND1 (blue) and SoxND2 (green) ChIP datasets. SoxN core binding intervals are displayed in black, matches to the SoxN binding motif as thin bars and the locations of know cis-regulatory modules (CRMs) as grey bars. SoxN binding in the gsb-n and gsb (top panel), nerfin-1 (middle panel) and pdm2 (bottom panel) regions are displayed. 
at each time point. This led to the identification of genes up- and downregulated in the mutants across the whole time course, as well as a third set of genes more variably expressed across the time course (Figure 1B). The enrichment in Gene Ontology biological process (GO:BP) terms in these three groups showed a marked difference (Figure S1A in Additional file 2 and Table S1D-I in Additional file 1). The 647 downregulated genes were the most relevant from a neural development perspective, being enriched in transcriptional regulation and specific terms related to early and late CNS development. This indicates that many of the genes directly or indirectly activated by SoxN are involved in controlling gene expression during neural development, in processes ranging from NB fate commitment through to neuronal development and differentiation. Conversely, while the list of 679 upregulated genes contained a few examples of genes known to play a role in the CNS, the list was enriched for very few nervous system GO terms but overrepresented for stress response terms, suggesting that at least some of the upregulated genes may represent a reaction to development in the absence of transcriptional regulators such as SoxN and its downstream targets. Finally, the set of variable genes, most of which were downregulated until stage 9, showed increased expression during stages 10 to 11 and returned to basal levels at the latest stages analysed, showed little significant GO enrichment, although we noticed several genes with known roles in CNS development (for example, beat-Ia, Fas3, frac, Kr-h1, lbl, Lim3). Overall, these data suggest that SoxN mainly functions in the nervous system as a transcriptional activator to promote the expression of both transcriptional regulators and effectors involved at all stages of neural development but may also act to repress some genes with CNS functions as well as more generic biological functions.

\section{A genome-wide view of SoxN binding}

To map high confidence SoxN binding intervals across the genome we employed two complementary approaches, DNA adenine methyltransferase identification (DamID) and chromatin immunoprecipitation (ChIP), using genomewide tiling arrays. We first used DamID to generate a reference profile of SoxN binding across stages 8 to 11 of embryogenesis (SoxNDam). Next, to provide independent validation of the DamID binding, we also produced a set of four ChIP datasets, employing three different antisera. Two of the antisera (SoxND1 and SoxND2) were used to generate SoxN ChIP profiles across the same developmental stages as the DamID experiment. We also generated a new affinity purified antiserum (SoxNPA179), showing consistent SoxN expression by whole-mount immunohistochemistry, and used this to create two further datasets (SoxNPA179 Early, stages 7 to 10, and SoxNPA179 Late, stages 11 to 13$)$.
All of the DamID and ChIP data were similarly processed and bound regions were identified according to a false discovery rate (FDR) model (Table 1A). Based on the smoothed window score profiles and the number of binding intervals detected, we focused on stringent FDR $1 \%$ data from the SoxNDam and the SoxND1 and SoxND2 ChIP experiments. In the case of the SoxNPA179 ChIP datasets, we reasoned that the narrower time windows employed could restrict the identification of comparable binding intervals and we selected the FDR 5\% datasets for further analysis (Table 1B). We compared the binding intervals and associated genes from DamID and ChIP assays (Figure 1C; Figure S1B,C in Additional file 2) and found a general concordance between the datasets. We then combined the five datasets to generate a core set of SoxN binding intervals that we used for further analysis. Since we only selected binding intervals with supporting DamID and ChIP evidence, this is a conservative approach and it is likely that SoxN interacts with a larger fraction of the genome than we report here.

These combined DamID and ChIP data identified 5,482 SoxN binding intervals associated with 3,251 genes, enriched for GO:BP terms relating to general and nervous system development, as well as RNA transcription and regulation (Table S2A-C in Additional file 3). To support the reliability of our analysis, we assessed the overlap between our SoxN-bound genes and those identified in a previously published small-scale ChIP analysis of SoxN binding [50]. Of 26 SoxN-bound genes identified in this study, 18 are present in our core binding interval set, a further 4 showed evidence of SoxN binding but below our threshold and only 4 were negative in our assays. Looking at the general properties of SoxN binding intervals, we found they are often in close proximity to transcription start sites (TSSs; Figure S2A in Additional file 4); however, there does not seem to be preferential binding of SoxN

Table 1 SoxN binding datasets, intervals and genes

\begin{tabular}{lcccc}
\hline A & FDR 1\% & FDR 5\% & FDR 10\% & FDR 25\% \\
\hline SoxNDam & 6,518 & 11,133 & 14,223 & 19,186 \\
SoxND1 & 5,830 & 11,988 & 16,786 & 27,272 \\
SoxND2 & 5,599 & 7,904 & 9,650 & 13,424 \\
SoxNPA179 Early & 3,145 & 6,335 & 9,001 & 15,818 \\
SoxNPA179 Late & 1,556 & 3,502 & 5,348 & 10,437 \\
\hline B & & Intervals & \multicolumn{2}{c}{ Genes } \\
\hline SoxNDam FDR 1\% & & 6,518 & \multicolumn{2}{c}{3,557} \\
SoxND1 FDR 1\% & & 5,830 & \multicolumn{2}{c}{4,212} \\
SoxND2 FDR 1\% & & 5,599 & \multicolumn{2}{c}{4,073} \\
SoxNPA179 Early FDR 5\% & 6,335 & \multicolumn{2}{c}{4,529} \\
SoxNPA179 Late FDR 5\% & 3,502 & \multicolumn{2}{c}{2,652} \\
\hline
\end{tabular}

(A) Number of intervals retrieved by the peak calling algorithm for the five binding datasets at different FDRs. (B) Number of genes associated with each high confidence binding dataset. 
upstream of the TSS, since the fraction of intervals mapping upstream (47.6\%) or downstream (52.3\%) is comparable. Interestingly, Sox 2 binding in the vicinity of TSS has also been reported [51]. We used the midpoint of each binding interval to assess the genomic features associated with SoxN binding and found a high proportion mapping to genic $(66.8 \%)$ rather than intergenic (33.2\%) regions. Notably, within genes, we found that introns $(25.8 \%)$ were more targeted than exons (17.7\%) and that UTRs accounted for only $4.7 \%$ of intervals (Figure $2 \mathrm{~A}$ ). The binding intervals were divided into the three main categories intergenic, intronic and exonic, and gene lists were generated

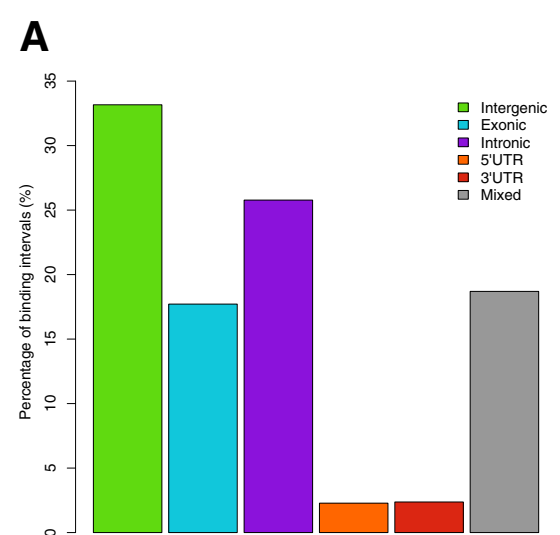

B

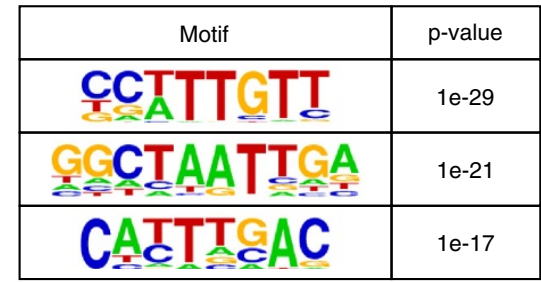

C

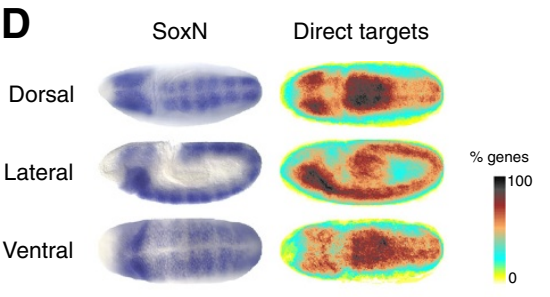

\section{E}

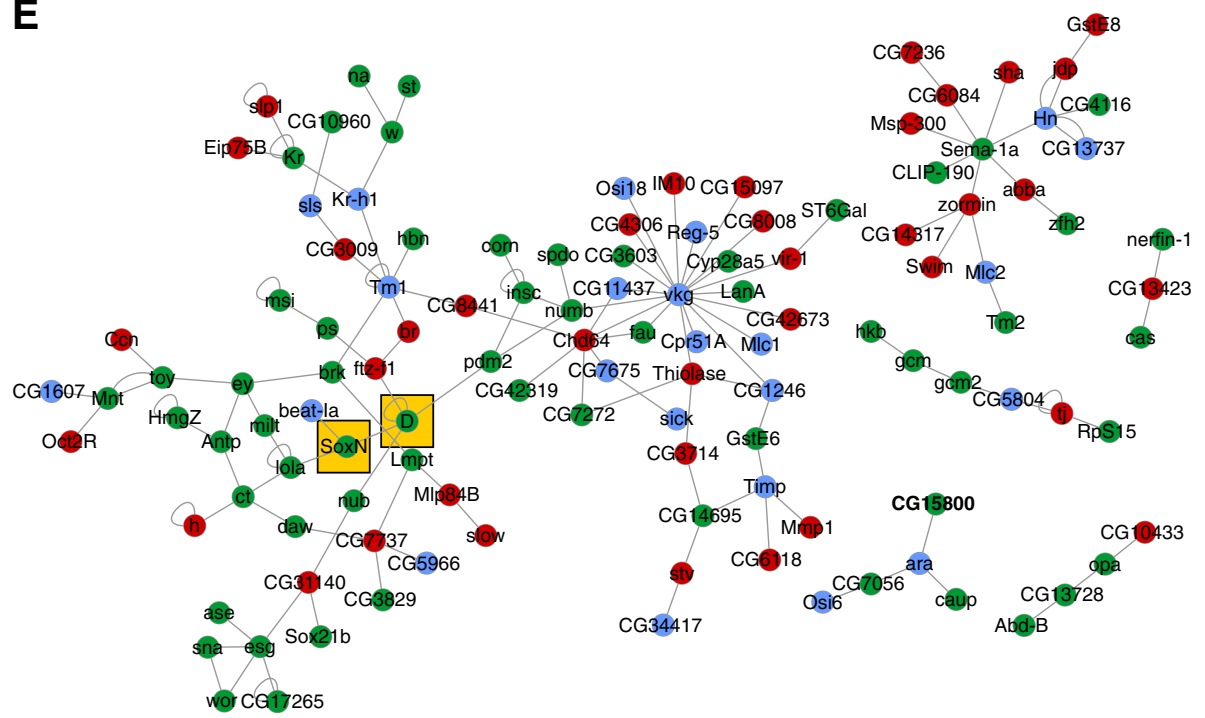

Figure 2 Features of SoxN binding and SoxN direct targets. (A) Barplot representing genomic features hit by SoxN binding intervals. 'Mixed' indicates intervals hitting more than a single feature in different genes. (B) De novo motifs discovered in the SoxN core dataset. The top three motifs found and their associated $P$-values are reported. (C) Proportional Venn diagram showing the overlap between genes differentially expressed in SoxN mutants (green, left) and genes bound by SoxN (blue, right). (D) Dorsal, lateral and ventral views of SoxN expression in a stage 9-10 embryo (left, images from the Berkeley Drosophila Genome Project) and false-colour heatmaps representing the average expression pattern of SoxN direct targets at the same developmental stages. (E) Network showing known interactions between SoxN direct targets obtained by superimposing the list of SoxN direct targets with a network created from the DrolD database. Genes are colour-coded according to their expression trend during the time-course (green, downregulated; red, upregulated; light blue, variable). SoxN and Dichaete are highlighted with yellow boxes. 
for each set (Table S2D-I in Additional file 3). Remarkably, The GO:BP enrichment computed for each of the resulting gene lists showed considerable differences (Figure S2B in Additional file 4; Table S2J-L in Additional file 3). Intergenic hits were highly enriched in processes related to the regulation of transcription and gene expression, while intronic hits had a clear developmental signature containing terms related to neurogenesis and morphogenesis. The level of enrichment found for exonic hits was substantially lower than those observed for the two other categories and only featured generic GO:BP terms.

We examined the sequence composition of SoxN binding intervals and noticed a marked increase in the average GC content at the centre of the intervals compared to the flanking $10 \mathrm{~kb}$ (Figure $\mathrm{S} 2 \mathrm{C}$ in Additional file 4). The GC content profile mirrored the average phastCons score distribution calculated from multiple genome alignments of $D$. melanogaster with 14 other insect species (Figure S2D in Additional file 4), showing that core SoxN binding intervals are well conserved. Sox domains bind to a conserved DNA motif (5'-WTTGWW-3') $[52,53]$, and scanning the core binding intervals with positional weight matrices from different species revealed high scoring matches to known Sox motifs (Figure S2E in Additional file 4). Similarly, performing a de novo motif discovery search identified a top-scoring motif closely matching the Sox consensus (Figure 2B). Similar, but not identical, motifs are reported for Dichaete [54,55]. We mapped high scoring matches $(P<1 \mathrm{E}-4)$ to the new SoxN motif across the genome [56] and identified over 43,000 matches, displayed as tracks in Figure $1 \mathrm{C}$ and subsequent binding profile figures, that show a good correspondence with the binding intervals we selected. The second and third highest scoring motifs identified in the de novo search are similar to homeobox binding sites. In particular, we note that motif 2 is very similar to those reported for $\mathrm{Dr}$ and Ind [54], while motif 3 closely resembles that of Vnd [57], three proteins playing key roles in the specification of neural identity across the DV axis. Thus, our analysis reveals a core set of well-conserved SoxN binding intervals, enriched for a novel SoxN binding motif, along with motifs associated with other TFs involved in Drosophila CNS development.

We compared our core SoxN binding intervals with binding intervals and enriched chromatin domains reported by the Berkeley Drosophila Transcription Network Project (BDTNP) [58,59] and the Model Organism Encyclopedia of DNA Elements (modENCODE) [60,61]. We found highly significant (z-score > 200) overlaps between binding intervals for SoxN and several TFs, including a number known to be involved in aspects of embryonic nervous system development $(\mathrm{Hb}, \mathrm{Kr}$, Dichaete, Med, Sens and Da). Many of the genes for these TFs ( $\mathrm{Hb}$, $\mathrm{Kr}$, Dichaete and Med) contain SoxN binding intervals, suggesting that SoxN may regulate as well as interact with them during CNS development. As expected, the profile of SoxN overlaps is very similar to those observed with other TFs involved in CNS development ( $\mathrm{Hkb}, \mathrm{Kr}$, Ubx and Zfh1; Figure S3A in Additional file 5). We also identified significant overlaps between SoxN binding and some histone-modifying proteins (particularly histone acetyltransferases and deacetylases) as well as domains enriched for several histone modifications. The majority of the histone modifications overlapping with SoxN binding are associated with active chromatin. However, we also found an association with histone marks normally associated with transcriptional silencing or repression, suggesting that SoxN may also act as a transcriptional repressor. Alternatively, this may highlight bivalent areas containing marks for both activation and repression that are poised for transcription [62], or it may simply reflect the fact that across the embryo some genes are repressed in particular cell lineages and active in others. As with the TF overlap, the pattern observed with SoxN is very similar to those observed with other nervous system regulators (Figure S3B in Additional file 5).

To link SoxN binding with mapped cis-regulatory modules (CRMs) in the Drosophila genome, we compared the core binding intervals with enhancer regions defined by REDFly (1,864 CRMs from 500 genes) [63] and FlyLight (7,113 CRMs from 970 genes) [64]. We found SoxN binding overlapping with 1,511 of 8,959 (17\%) unique CRMs defined by both databases, including 704 out of the 4,724 (15\%) FlyLight enhancers reported to show CNS expression (Table 2). Taken together, these observations support the general conclusion that SoxN acts as a transcriptional activator, interacting with other TFs at known CRMs, to control expression of a set of genes essential for CNS development.

\section{Identification of SoxN direct targets}

To uncover a high confidence set of SoxN target genes, we intersected the differential expression data (1,665 genes) and core SoxN binding intervals (3,251), identifying 536 genes that we assigned as direct SoxN targets. We added a further 7 genes that were not identified as SoxN bound because of the computational approach we used to assign intervals to genes, resulting in 543 targets (Figure 2C; Table S3A in Additional file 6). Of these, 199 genes were consistently downregulated, 213 upregulated and 131 variable in the microarray time course (Table S3B-D in Additional file 6). We emphasise this is a conservative estimate since our stringent selection criteria for binding and differential expression are likely to exclude many bona fide binding events and small, but functionally relevant, changes in gene expression. In addition, loss of SoxN binding at some genomic locations is likely to be rescued by Dichaete activity (see below). Despite these caveats, we 
Table 2 Overlaps between SoxNeuro core intervals and known cis-regulatory modules

\begin{tabular}{lccccc}
\hline & CRMs & $\begin{array}{c}\text { CRMs overlapping with } \\
\text { SoxN core intervals }\end{array}$ & Genes & $\begin{array}{c}\text { Genes overlapping with } \\
\text { SoxN core intervals }\end{array}$ & Genes in SoxN core \\
\hline REDfly & 1,864 & $492(26.4 \%)$ & 500 & $135(27.0 \%)$ & $218(43.6 \%)$ \\
FlyLight & 7,113 & $1,023(14.4 \%)$ & 970 & $342(35.3 \%)$ & $364(37.5 \%)$ \\
FlyLight CNS & 4,724 & $704(14.9 \%)$ & 780 & $267(34.2 \%)$ & $307(39.4 \%)$ \\
All (REDfly + FlyLight) & 8,959 & $1,511(16.9 \%)$ & 1,302 & $418(32.1 \%)$ & $477(36.6 \%)$ \\
\hline
\end{tabular}

Number and percentages of CRMs and associated genes from REDfly and/or FlyLight displaying overlap with SoxNeuro core intervals. 'FlyLight CNS' refers to FlyLight CRMs reportedly driving expression in the CNS. 'All' refers to the total number of unique CRMs in the REDfly and FlyLight sets combined together.

found that approximately a third of genes with significant expression changes in SoxN mutant embryos were also bound by SoxN, and that over $15 \%$ of SoxN-bound genes showed expression changes at our significance threshold. As expected, we found that the GO:BP enrichment was similar to that of the two original datasets, with development and transcription-related terms overrepresented (Table S3E in Additional file 6). Enrichment of more specific terms associated with NB specification and fate commitment, and a range of terms relating to the development of glia, neurons and their projections was also found.

We examined the embryonic expression patterns of the SoxN target genes using genome-wide expression maps [65], and found that the average expression of the target genes closely matches that of SoxN CNS expression, supporting the reliability of our dataset (Figure 2D). Using the DroID database [66], we rendered a network featuring all known high confidence Drosophila genetic and protein-protein interactions, and superimposed our list of SoxN targets onto this. All modules with more than two nodes were selected to retrieve the most significant known interactions between SoxN direct targets (Figure 2E). The resulting subnetworks are highly interconnected and contain many proteins involved in specific aspects of nervous system development such as asymmetric NB division (Insc, Numb, Spdo, Sna, Wor and Esg), gliogenesis (Hkb, Gcm and Gcm2) and eye development (Ey and Toy), as well as most of the TFs involved in the temporal progression of NB identity (Cas, D, Kr, Nub and Pdm2). We also identified proteins specifically involved in the development of neuronal projections (Ct, Daw, Nerfin-1 and Sema-1a), and a set of homeodomaincontaining proteins (Abd-B, Antp, Ara, Caup and Zfh2) with various roles in the CNS.

Our analysis indicated that SoxN directly regulates a large group of TFs and effectors with a range of diverse functions in CNS development as illustrated with a selection of genes taken from a clustering analysis (Figure 3A). To confirm this, 29 of the most functionally relevant genes, including 19 from the network described above, were selected for validation by immunohistochemistry or in situ hybridization. These included proneural genes, TFs controlling NB divisions and identity, as well as TFs involved in aspects of glial or neuronal differentiation such as axon fasciculation. Strikingly, the expression of all of these was disrupted in SoxN mutants, in many cases very severely (Figure 3B; Additional file 7). In particular, we frequently observed reduced and/or altered expression patterns in the most lateral domains of the neuroectoderm, where Dichaete is not expressed and therefore unable to functionally compensate for the loss of SoxN. We also examined the expression of a selection of these targets in embryos ectopically expressing SoxN via a $K r$-Gal4 driver (Figure 3B). We found that Ac, Ase, Cas, Dichaete, Pros and Wor expression was severely altered in SoxN misexpressing embryos, with increased and ectopic expression in the lateral domains of the neuroectoderm, supporting the view that our proposed targets are under direct SoxN transcriptional control. Analysis of genes not expected to be affected by loss or gain of SoxN (ind in the medial column of the neuroectoderm (Figure 3B), and sim in the midline (Figure S4A in Additional file 7)) indicate that the expression phenotypes we observe are not due to a general disruption in the organisation of the CNS. Overall, we have identified key roles for SoxN in all aspects of embryonic CNS development, and showed that it regulates sets of TFs and effectors involved in processes ranging from the earliest events in neural identity specification to the terminal differentiation of neurons and glia.

To relate the activity of SoxN to its mammalian orthologues, we compared the set of SoxN-bound genes with those identified as Sox2 or Sox11 targets in mouse. Bergsland and colleagues [48] identified 1,388 regions bound by Sox 2 in neural precursor cells that correspond to 1,100 genes. We mapped these genes to their Drosophila orthologues and found that 443 are conserved in our set of SoxN targets (Table S4A in Additional file 8). In other words, more than $40 \%$ of Sox2-bound genes are also bound by SoxN, but this core of conserved targets represents only approximately $13.5 \%$ of SoxN-bound genes, suggesting that SoxN has more diversified functions than Sox2 in the CNS. The list of shared targets is, as expected, enriched for TFs and effectors with roles in CNS development (Table S4B in Additional file 8), including the DV patterning homeobox genes $\mathrm{Dr}$ and $v$ d, proneural genes, bHLH genes in the Enhancer of split complex and many other transcriptional regulators whose absence is known to cause CNS phenotypes in both organisms. In the case 

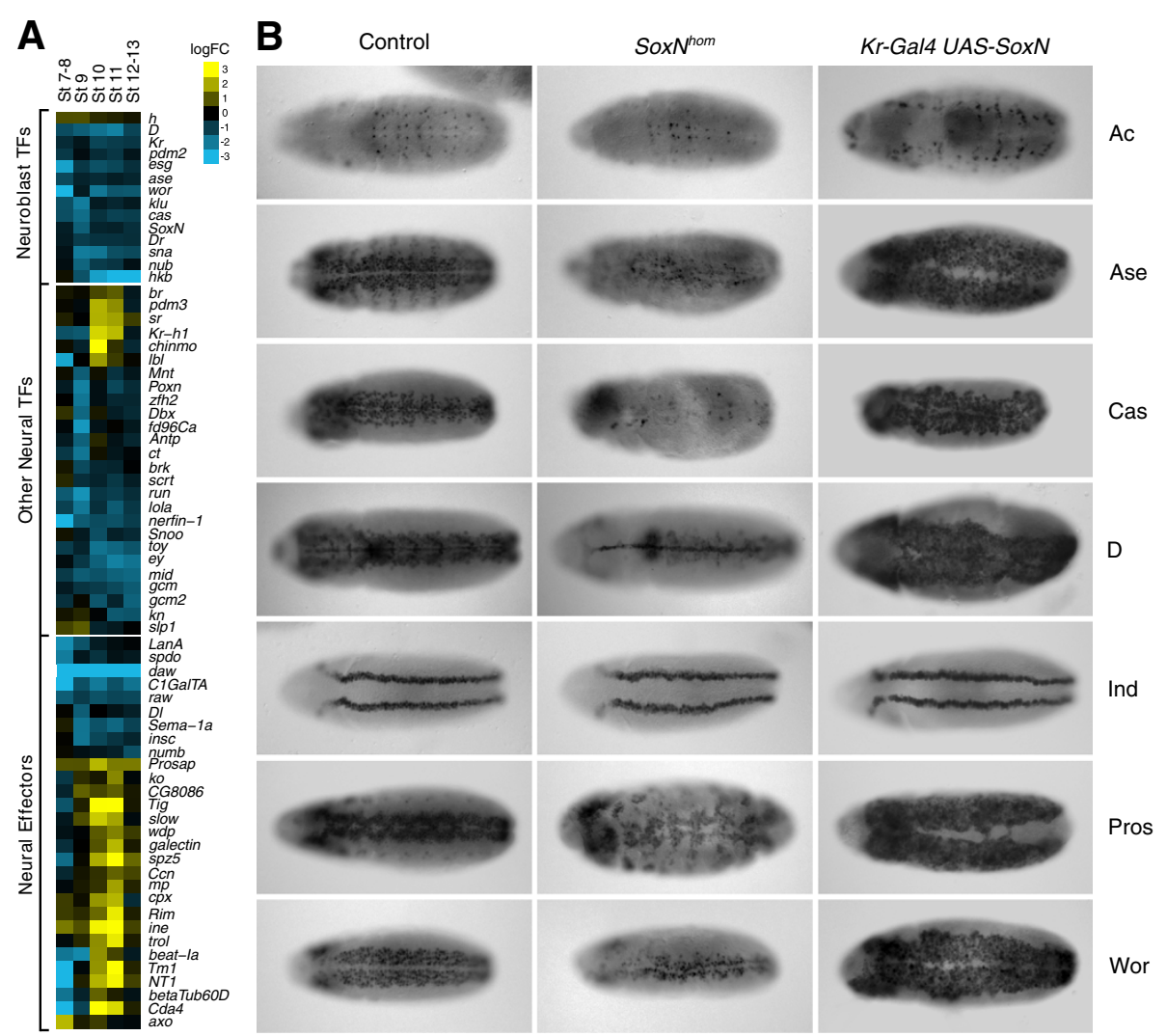

Figure 3 Validation of SoxN direct targets. (A) Selected genes from a hierarchical clustering of expression trends highlighting three functional groups of SoxN direct targets. $\log F C=\log _{2}$ fold change. (B) Immunohistochemical stainings of a selection of SoxN direct targets identified in this study in control embryos (left), SoxN homozygous mutants (middle) and Kr-Gal4 UAS-SoxN embryos (right). All embryos are shown as ventral views with anterior to the left. Ind, showing normal expression in mutant and transgenic embryos, is provided as a negative control.

of Sox11, a group C Sox protein involved in neural differentiation, we found a much larger overlap. Over a third of the SoxN bound genes (34\%, 1,092 genes) have mouse orthologues bound by Sox11 in neural precursors or differentiating neural cells (Table S4C in Additional file 8), including TFs and effectors with roles in both early neural specification and neuron differentiation (Table S4D in Additional file 8). We also identified 722 genes bound by SoxN and Sox11 but not Sox 2 (Table S4E in Additional file 8), which are enriched for terms related to neuronal projection development and morphogenesis (Table S4F in Additional file 8). Together, these observations suggest that the role of Sox proteins in neural development is highly conserved and, importantly, that SoxN regulates a set of target genes controlled by group B and group C Sox proteins in vertebrates.

\section{SoxN and Dichaete binding in Sox mutant embryos}

In both flies and vertebrates, group B Sox proteins are able to functionally compensate, with single gene mutants showing comparatively weak phenotypes in regions where related proteins are coexpressed. To gain a genomic perspective into this functional redundancy, we generated four additional DamID datasets, assaying the binding of SoxNeuro and Dichaete in wild-type and null mutant embryos lacking the other factor. We refer to these datasets as SoxNDam (SoxN binding in wild type), DDam (Dichaete binding in wild type), D-SoxNDam (SoxN binding in Dichaete mutants) and SoxN-DDam (Dichaete binding in SoxN mutants). We used null alleles of both SoxN and Dichaete for the analysis. SoxN $N^{U 6-35}$ has a premature stop codon before the DNA binding domain and is a protein null [31,32]. The Dichaete ${ }^{r 72}$ allele has not been molecularly defined but, genetically, it behaves as an amorph in all phenotypic assays [30]. The experiments were performed with hand-picked embryos selected between stages 12 and 17 of embryogenesis to allow sufficient time for the expression of the yellow fluorescent protein (YFP) marker used to identify homozygous mutants. It should be noted that these datasets differ from the SoxN data described above since profiles were generated from non-overlapping stages of development and utilised much smaller sample sizes. While the binding we map in this comparative experiment is not directly comparable with our defined SoxN core dataset or with our previous work defining Dichaete binding, we note that 
after stage 11 there is still substantial expression of both proteins in the CNS, particularly in the brain and in late segregating trunk neuroblasts, GMCs and their progeny $[33,35-37,50]$. In addition, post-mitotic cells, such as neurons and glia, expressing the Sox-Dam fusions prior to stage 12 will also be identified in this analysis due to perdurance of the adenine methylation mark.

Comparing genome-wide profiles by simply overlapping the genomic coordinates of peaks called individually for each dataset is a rather coarse approach and can potentially underestimate binding similarity [67]. In simple pairwise comparisons, peaks with similar height and area may be called in one sample but not the other because of the fixed thresholds applied to each dataset by peak calling algorithms, thus limiting meaningful comparison of binding profiles in different conditions. To overcome this issue, we developed a method to directly compare the normalised ratios of each microarray probe and compute similar and dissimilar genomic regions. We named this tool SimBindProfiles [68] and used it to perform pairwise comparisons between the four datasets and uncover similarly or differentially bound regions (Figure 4A-C). While SimBindProfiles identifies genomic regions that are similar or dissimilar between the profiles being compared, its output is not directly comparable with the binding intervals identified by threshold-based peak calling algorithms. Table 3 summarises the numbers of genomic regions and associated genes obtained with the analysis; all of the corresponding genomic regions, gene sets and corresponding GO:BP enrichments are provided in Additional file 9.

The genome-wide binding profiles of SoxN and Dichaete in wild-type embryos showed extensive overlap (2,893 regions, 1,890 genes), indicating that the proteins often bind at the same locations (Figure 4D). Consistent with their biological roles, the set of common bound genes were enriched for developmental, CNS and transcriptional regulation GO:BP terms (Additional file 10). The set includes major regulators of early CNS specification, including the proneural genes of the achaete-scute complex, the DV patterning TFs encoded by $D r$ and $v n d$, and the NB temporal identity genes (svp, $h b, k r$ and $p d m 2$ ). Altogether, we found that both Sox proteins commonly bound to over a hundred genes encoding TFs with roles in a range of CNS processes. We also identified a large number of genomic regions uniquely bound by either SoxN (Figure 4E; 3,723 regions, 1,649 genes) or Dichaete (Figure 4F; 3,506 regions, 1,753 genes), indicating that their binding pattern is not fully redundant and that they exert at least some of their functions independently of one another. While the gene set uniquely bound by Dichaete was also enriched for GO:BP terms relating to development, CNS functions and transcription, the SoxN unique gene set showed comparatively weak enrichments, although it does contain a set of 95 genes annotated with neuronal differentiation functions (Additional file 10). Thus, Dichaete and SoxN share a common set of targets involved in early and late CNS development. A set of genes with similar functions are uniquely regulated by Dichaete, whereas SoxN unique targets appear to be downstream effectors of basic cellular processes, perhaps indicative of a role in terminal differentiation.

To directly address functional redundancy, we examined the binding profiles of SoxN and Dichaete in embryos homozygous for null mutations in the other protein (Figure 4B,C) and identified five different types of event: 1) no change-the binding of each protein was not affected by the loss of the other; 2) compensation - one Sox protein compensated for the loss of the other by binding at locations normally occupied by the latter (Figure $5 \mathrm{~A}$ ); 3 ) increased binding-in the absence of one Sox protein, the other showed an increase in binding at its normally occupied intervals (Figure 5B); 4) de novo binding-in the absence of one Sox protein, the other bound at new regions not normally bound in the wild type (Figure 5C); 5) loss of binding - lack of one Sox protein resulted in loss of binding of the other (Figure 5D).

While at a global level SoxN binding was broadly similar in wild type and Dichaete mutants (3,720 regions), a detailed examination identified a variety of changes. In 794 instances SoxN compensated for the loss of Dichaete and increased binding events were identified at 245 genomic locations. SoxN was also often found to bind at new, previously unbound, locations (1,893 instances), but the scenario with the highest impact was loss or strong reduction of binding (2,497 regions), suggesting that Dichaete is often required for the recruitment or retention of SoxN. A considerable proportion (30\%, 536 genes) of the 1,753 genes uniquely bound by Dichaete showed evidence of compensation by SoxN at the genic level (considering both compensation and de novo but not increased binding events) and these were primarily genes annotated with CNS functions and transcriptional regulation. Interestingly, the majority ( $58 \%, 896$ genes) of the 1,539 genes that showed a loss of SoxN binding were genes uniquely bound by SoxN. The gene sets for all types of event were associated with moderate to high overrepresentation of GO:BP terms related to CNS development and transcriptional regulation (Figure S6A in Additional file 11).

In contrast, we found that Dichaete binding was less affected by the loss of SoxN. We observed no change in Dichaete binding in SoxN mutant embryos at 5,175 regions, while the other scenarios were observed at much lower frequencies. Dichaete was found to compensate for loss of SoxN at only 276 locations and showed increased binding at 102. De novo and loss of binding (658 and 943 instances, respectively) were also similarly reduced. All the associated gene sets were somewhat less 

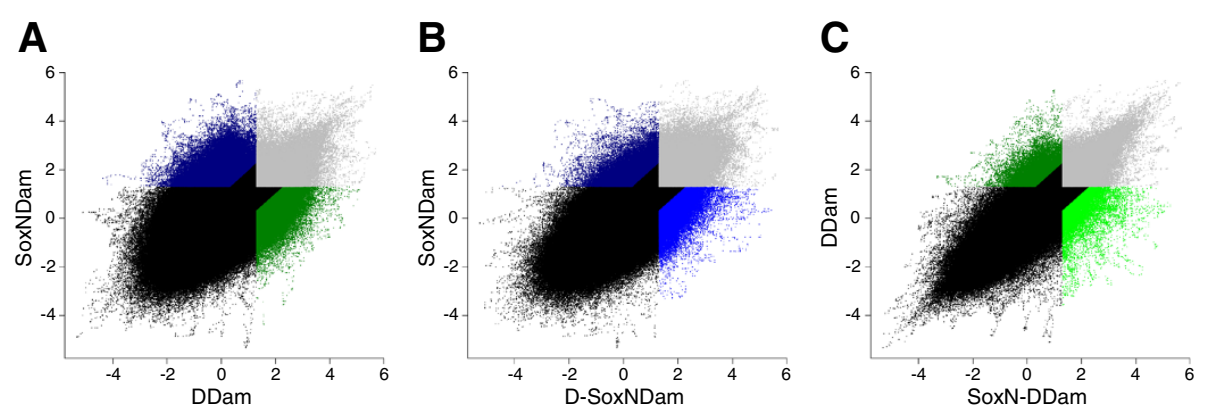

D

SoxNeuro and Dichaete common binding

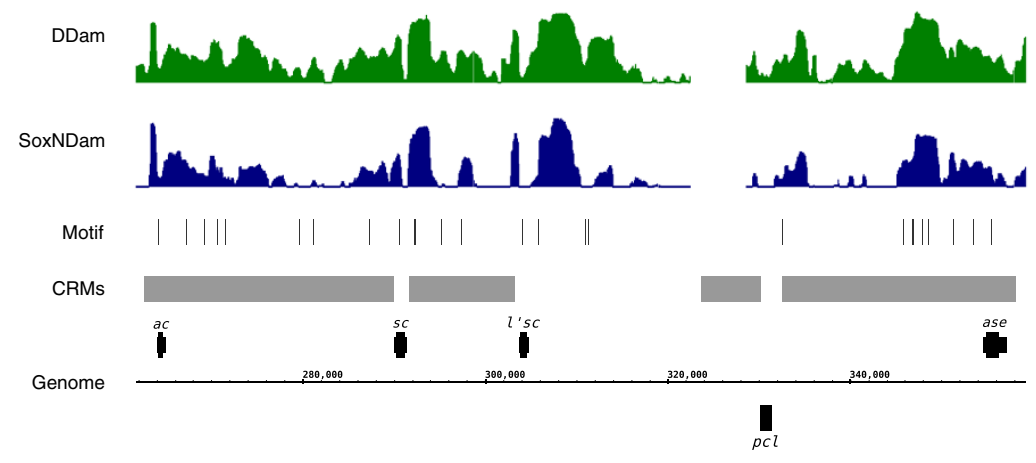

E

SoxNeuro unique binding

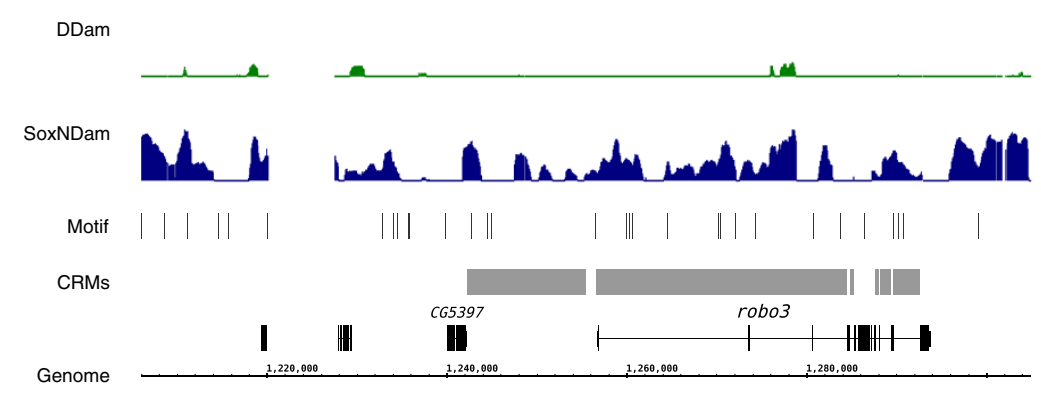

$\mathbf{F}$

Dichaete unique binding

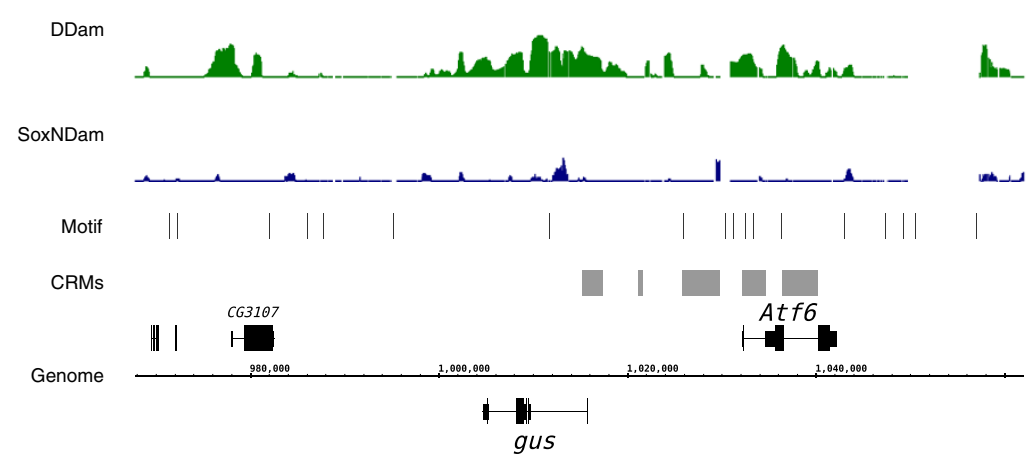

Figure 4 (See legend on next page.) 
(See figure on previous page.)

Figure 4 SoxN and Dichaete differential binding. (A-C) Differential binding in pairwise comparisons of the SoxNDam, DDam, D-SoxNDam and SoxN-DDam datasets as normalised probe intensities ( $\log _{2}$ fold change). Light grey areas are probes bound in both datasets, black regions are not bound in either. (A) SoxNDam (dark blue) and DDam (dark green); (B) SoxNDam (dark blue) and D-SoxNDam (light blue); (C) DDam (dark green) and SoxN-DDam (light green). (D-F) Representative SoxN and Dichaete binding profiles in wild-type embryos (dark blue and dark green, respectively). Matches to the SoxN binding motif are displayed as thin bars, FlyLight and REDfly enhancers are displayed in light grey. (D) SoxN and Dichaete common binding across the achaete-scute complex. (E) SoxN unique binding in proximity of robo3. (F) Dichaete unique binding in the gus and Atf6 region.

enriched for CNS development and gene regulation than their SoxN counterparts (Figure S6B in Additional file 11). Of the 1,649 genes uniquely bound by SoxN, only $14 \%$ (232 genes) showed evidence of Dichaete compensation (considering compensation and de novo binding events) and these were only weakly enriched for generic GO:BP terms. Finally, we examined the overlap with FlyLight CNS enhancers [64] and found that the number of enhancers bound by both SoxN and Dichaete (621 enhancers, corresponding to 237 genes; Table S6A in Additional file 12) was comparable to the number of enhancers hit by SoxN alone (623 enhancers, 238 genes; Table S6B in Additional file 12) or by Dichaete alone (704 enhancers, 258 genes; Table S6C in Additional file 12), reinforcing the idea that the two factors work independently as well as in concert to direct gene expression in the CNS.

Our analysis of the genome-wide binding comparison of paralogous TFs under mutant conditions strongly supports the hypothesis that group B Sox proteins have both independent and shared functions under normal conditions but can functionally compensate by occupying vacant binding sites when one of the proteins is absent. Importantly, our observations indicate that a considerable fraction of the redundant CNS functions is centred on a

Table 3 SoxN and D binding in wild-type, $D$ and SoxN mutant embryos

\begin{tabular}{lcc}
\hline Binding & Intervals & Genes \\
\hline SoxNeuro Dichaete common & 2,893 & 1,890 \\
SoxNeuro unique & 3,723 & 1,649 \\
Dichaete unique & 3,506 & 1,753 \\
SoxNeuro no change & 3,720 & 2,063 \\
SoxNeuro compensatory & 794 & 570 \\
SoxNeuro increased & 245 & 195 \\
SoxNeuro de novo & 1,893 & 1,113 \\
SoxNeuro loss & 2,497 & 1,593 \\
Dichaete no change & 5,175 & 2,868 \\
Dichaete compensatory & 276 & 226 \\
Dichaete increased & 102 & 87 \\
Dichaete de novo & 658 & 522 \\
Dichaete loss & 943 & 705 \\
\hline
\end{tabular}

Numbers of intervals retrieved by SimBindProfiles and associated genes for the SoxNDam, DDam, D-SoxNDam and SoxN-DDam datasets. core of TFs involved in aspects of neural specification and differentiation, suggesting that both Sox proteins have been maintained in the CNS to provide a degree of robustness to the regulatory networks driving early neurogenesis. Finally, the fact that SoxN targets in Drosophila and Sox 2 targets in mouse neural cells are well conserved emphasises that SoxB gene functions are essential in the regulatory networks underpinning the most basic aspects of neural development across metazoa.

\section{Discussion}

In this study we performed a genome-wide analysis of the role of the group B Sox gene SoxN during Drosophila embryonic development and generated a genomic perspective on the functional redundancy of Sox TFs. We identified a high confidence list of SoxN target genes that places SoxN at the heart of the regulatory networks driving neural specification and differentiation. We show an extensive overlap between SoxN and Dichaete genomic binding, but also identify binding indicative of unique functions for each $\mathrm{TF}$ during CNS development. In addition, we uncovered unexpected complexity in the relationship between SoxN and Dichaete, with evidence for compensation, dependency and other effects that can potentially explain why the coexpression of group B paralogs has been maintained throughout evolution. The fact that many SoxN targets have orthologs that are targets of Sox2 in mouse NSCs suggests that the roles of group B proteins in the CNS are well conserved. The underlying regulatory networks driving early myogenesis [69], as well as heart [70] and eye development [71], are known to be conserved, and it is likely that more of the core circuitry underpinning basic developmental processes has been maintained throughout animal evolution [72]. Together with the evidence that mammalian group B Sox proteins are able to rescue SoxN and Dichaete mutant phenotypes $[30,31]$, our data suggest an underlying conservation in the regulatory networks driving early aspects of CNS development across higher metazoans. In addition, the high overlap between SoxNeuro and Sox11 targets suggest SoxNeuro is also involved in late aspects of neural development and differentiation.

As with many other developmentally important TFs, we found that SoxN binds extensively across the genome, and a significant proportion of genes in the genome are 


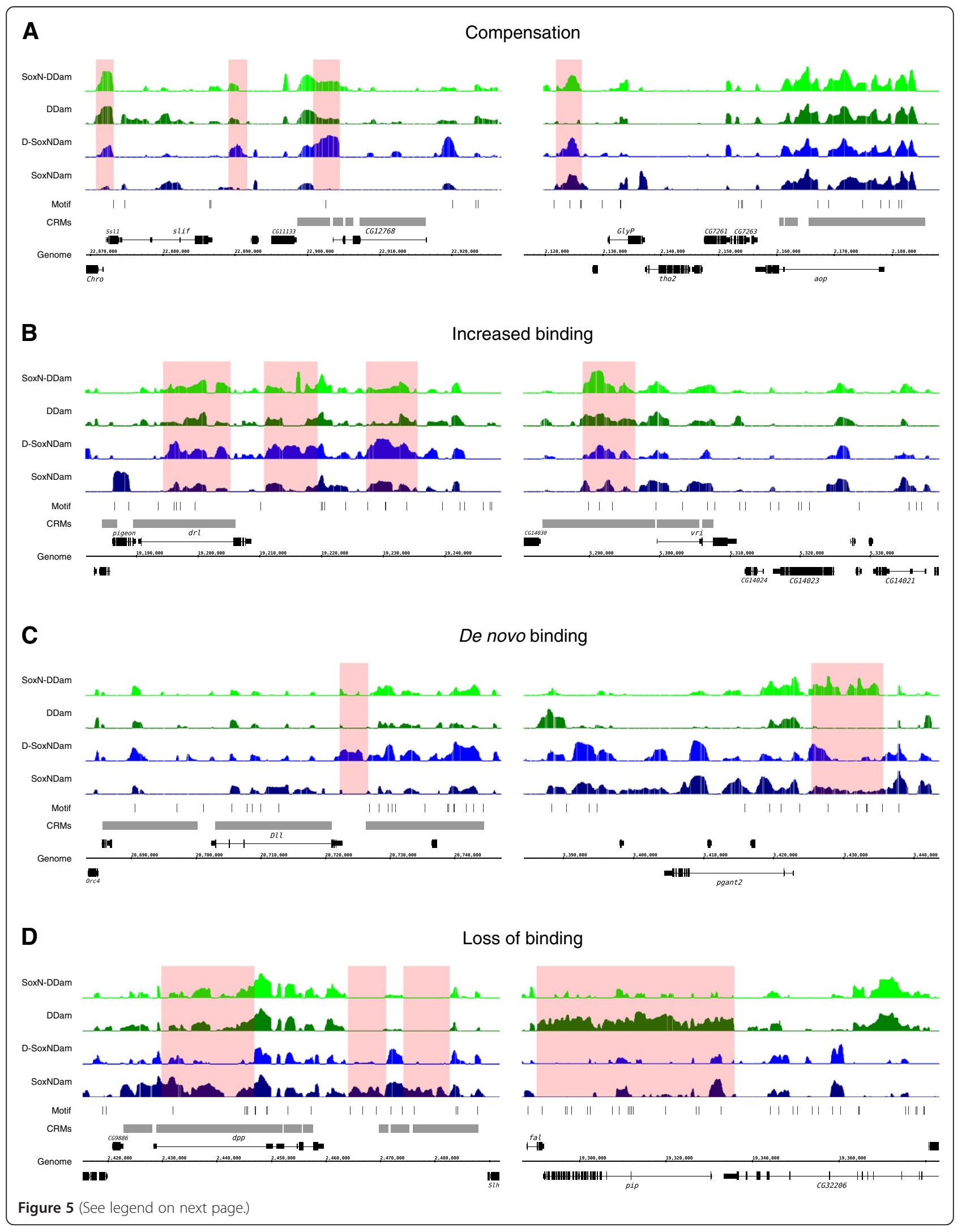


(See figure on previous page.)

Figure 5 Profiles of SoxN and Dichaete binding in Dichaete and SoxN mutant embryos. Representative SoxN binding profile in Dichaete mutant embryos (light blue) and Dichaete binding profile in SoxN mutant embryos (light green). Matches to the SoxN binding motif are displayed as thin bars, FlyLight and REDfly enhancers are displayed in light grey. Events of (A) compensation, (B) increased binding, (C) de novo binding and (D) loss of binding are highlighted as red shaded boxes.

affected by its loss. However, many of the genes misregulated in SoxN mutants may not be directly controlled by SoxN, but by regulators whose expression is dependent upon SoxN. Consistent with this, we found that many TFs involved in different aspects of neurogenesis and gliogenesis are downregulated in SoxN mutants, indicating that a prominent function of SoxN is to promote the expression of genes required for neural development. Some of the genes bound by SoxN may not show significant changes in their expression levels due to functional compensation by Dichaete and thus the network of Sox-related nervous system genes is likely to be even larger. In support of this view, we identified considerable overlap between SoxN and Dichaete binding across the genome, particularly at a number of genes with transcriptional roles in early aspects of neural development, as well as direct evidence of substantial Dichaete compensatory binding in SoxN mutants.

Focusing on what we believe to be unambiguous SoxN targets, genes that are both bound by SoxN and change expression in the mutant, we identified a set of genes involved in multiple aspects of embryonic development and morphogenesis. As expected, many of the targets have identified roles in CNS development and form a highly interconnected network, emphasising that SoxN regulates a range of processes, characterized by specific sets of target genes. We can broadly divide SoxN functions into two main categories: early in nervous system development, SoxN controls a battery of genes required for the correct specification of NBs, while at later stages it is involved in regulating the differentiation of both neurons and glia into mature, terminally differentiated cells. The involvement of SoxN in the specific regulation of terminal differentiation is supported by a previously reported analysis [50] that showed both SoxN binding at a set of genes involved in axonal pattering and genetic evidence that SoxN function is directly required for correct axonal pattering.

In particular, early in development SoxN promotes the expression of proneural genes $a c$ and ase while repressing the expression of hairy, a known proneural gene repressor [73], thereby driving the acquisition of the neural fate. Of note, SoxN and Dichaete display opposite behaviours during this initial stage of neural specification, since both $a c$ and ase are partially repressed by Dichaete [32,38]. Dichaete and SoxN interact with the homeodomain proteins Ind and Vnd, which specify neural identity across the DV axis [38,74]. We identified extensive SoxN binding at FlyLight enhancers associated with Vnd, as well as Dr, Egfr and Dichaete, other components of this developmental pathway, and observe changes in Dichaete and Dr expression in SoxN mutants. Since Dichaete also displays widespread binding at these DV patterning genes $[38,55]$, it is likely that Dichaete and SoxN act redundantly in this context. The loss of Dr expression in SoxN mutants is consistent with this idea, since Dr is restricted to the lateral column of the neuroectoderm where Dichaete is not expressed. In addition, the de novo motif discovery search we performed with SoxN binding intervals recovered motifs resembling those reported for Ind/Dr and Vnd [54,57]. We identified over 200 locations in the fly genome containing combinations of Sox and DV patterning TF binding motifs, including regions overlapping 68 FlyLight neural enhancers. In particular, we found co-occurrence of SoxN and Ind/Dr motifs at 43 FlyLight enhancers associated with early neural TF genes (Dichaete, $D r$, svp, pros and $\mathrm{g} \mathrm{cm}$ ). Together, these data strengthen the view that SoxN, Dichaete and the DV patterning homeodomain TFs interact at regulatory elements in the fly genome to drive establishment of neural fate $[33,38]$. Since a set of homeodomain proteins also cross-regulate to pattern the vertebrate neural tube and are coexpressed with group B Sox proteins [75], our observations support the view that the DV neural pattering regulatory network has been conserved across evolution [76] and indicate a crucial role for group B Sox proteins in this key aspect of early CNS specification.

Our analysis indicates that the role of SoxN in CNS development extends well beyond early specification events. We identified all the known components of the temporal cascade of TFs regulating neural identity as SoxN targets ( $h b, K r, n u b, p d m 2$, cas and $s v p)$. We also found highly significant overlaps between SoxN, Dichaete, $\mathrm{Hb}$ and $\mathrm{Kr}$ binding across the genome, suggesting the possibility of a regulatory feedback network where SoxN promotes the expression of temporal identity factors and then binds with them to orchestrate the differentiation of NBs. Dichaete also shows extensive binding at the genes in the temporal cascade [49] and, consistent with functional redundancy, we found $\mathrm{Cas}, \mathrm{Hb}, \mathrm{Kr}, \mathrm{Nub}$ and $\mathrm{Pdm} 2$ expression primarily affected in the lateral column of the neuroectoderm in SoxN mutants, where Dichaete is not expressed. As we note above, Dichaete has been shown to function in this regulatory cascade [42,77], indicating that group B Sox proteins generally participate in the regulatory networks generating neuronal diversity. We also identified and validated targets implicating SoxN in the regulation of genes controlling self-renewal and asymmetric 
divisions of NBs and their progeny, ganglion mother cells (insc, numb, spdo, sna, wor and esg [78-84]), and have previously identified roles for Dichaete in these pathways [49]. We note that in vertebrates, B1 proteins are involved in the control of NSC self-renewal and must be downregulated to allow neural differentiation [20-22], further emphasising similarities between fly and vertebrate SoxB functions.

Finally, we identified a substantial number of SoxN targets with known functions in the development and morphogenesis of neuronal axons and dendrites, including ct [85], daw [86,87], Dbx [88], kn [85], lola [89], mid [90], nerfin-1 [91] and Sema-1a [92], thus implicating SoxN in the direct regulation of genes involved in terminal neural differentiation. Our observations support a previous analysis that demonstrated SoxN is expressed in a subset of postmitotic neurons and glia, binds at genes involved in late aspects of neural differentiation and shows axonal phenotypes when mis-expressed or in genetic interactions with its targets (lola and beat1a) [50]. In addition, in several cases (daw, Dbx, lola, mid, nerfin-1 and Sema-1a), mutant phenotypes have been described for SoxN targets that show striking similarities to the lateral axonal phenotypes of SoxN mutants [32]. Similarly, we found that SoxN regulates the expression of $\mathrm{gcm}$ and $\mathrm{gcm} 2$, the two TFs responsible for the specification and differentiation of all Drosophila glial cells [93-95]. We also found that SoxN activates $h k b$, which has been reported to physically interact with $\mathrm{Gcm}$, triggering its autoregulation [96]. Together with the glioblast defects reported in SoxN mutants [32], these observations strongly link SoxN to gliogenesis.

Our findings highlight a major difference in the roles group B Sox proteins play in fly and vertebrate CNS development. In vertebrates, the B group has evolved two subclasses, each with specialised and restricted functions: SoxB1 proteins are required for the maintenance of neural precursors, whereas SoxB2 proteins counteract their action, promoting cell cycle exit and neural fate commitment. Differentiation into mature neural cells is promoted by other groups of Sox TFs, primarily groups C (Sox4, Sox11 and Sox12) and E (Sox8, Sox9 and Sox10) $[20,97]$. In contrast, our data suggest a simpler system in insects, where SoxN and Dichaete are the only Sox genes contributing to the majority of the processes in embryonic neurogenesis, and are reused in different contexts during CNS development. Of the remaining six Sox genes in the fly genome, only the group B gene Sox $21 a$ and the group $\mathrm{D}$ gene Sox $102 \mathrm{~F}$ show detectable expression in the embryonic CNS, but in both cases expression is relatively late in development and restricted to a handful of specific cells [27]. Thus, in Drosophila, all aspects of CNS development, from neural specification through to terminal differentiation, are under the control of group B Sox proteins. The view that SoxB proteins have evolved different roles in insects and vertebrates while maintaining their core functionality is supported by the comparison of gene sets bound by SoxN in Drosophila and Sox 2 or Sox 11 in mouse, which indicates more diversified functions for SoxN in the CNS. Core regulatory genes involved in neural specification and NSC biology are targets of SoxN and, while a set of later target genes involved in neural differentiation are shared by SoxN and Sox11. We also identify a number of intriguing similarities between the roles of Sox proteins in flies and vertebrates during neural development. The contrast between the roles of Dichaete and SoxN in the regulation of proneural genes, with SoxN activating and Dichaete repressing, is reminiscent of the opposing functions shown by vertebrate SoxB1 and SoxB2 subgroups in NSC differentiation [22], and may point to the origin of the group B neofunctionalisation. Similarly, the two SoxB TFs display opposite activity in the regulation of pros, with SoxN acting as a transcriptional activator (this study), and Dichaete as a repressor [98]. Aside from these two specific examples, it appears that both SoxN and Dichaete mainly act as partially redundant activators with overlapping roles in early neural specification.

The mechanisms underlying why evolution has maintained substantial overlapping expression of closely related group B Sox proteins has so far remained elusive. While the binding patterns of SoxN and Dichaete in wildtype embryos look broadly similar, supporting a simple model where the two factors act redundantly, a large number of genomic locations display unique SoxN or Dichaete binding. SoxN unique genes appear to be associated with general cellular processes, possibly reflecting a role in terminal differentiation. In the case of Dichaete, we identified a set of uniquely bound TF genes likely to be linked to its roles in segmentation, early midline development and hindgut morphogenesis [34,35]. In line with this, we have also found the expression of many more genes affected in Dichaete than in SoxN mutant embryos [49]. Since genes showing substantial binding overlap are associated with regulatory networks driving early neural specification, we suggest coexpression has been maintained to provide a degree of robustness to these critical pathways that establish the foundations for early nervous system development. On the other hand, the different binding profiles of Drosophila group B paralogs we report here can be interpreted as examples of neofunctionalization.

The analysis of SoxN and Dichaete binding in their respective mutants provided molecular evidence to support the idea that each protein can functionally compensate for the loss of the other. Of interest, we found that SoxN was more able to substitute for Dichaete than vice versa. In some instances, we could explain a lack of compensation by the fact that each of the proteins has unique expression 
domains; however, since the DamID profiling method we employed to map binding events in the mutants relies on ubiquitous low level expression, lack of coexpression may not be a sufficient explanation. It is possible that regions that do not show compensatory binding reflect SoxN- or Dichaete-specific interactions with cofactors that are not shared between the paralogs, pointing to another level of neofunctionalization. In this respect, we note that SoxN has a role in cuticle patterning that is only partially compensated by Dichaete $[99,100]$, and some of the genes uniquely bound by SoxN have annotated roles in cuticle development. We also have preliminary evidence from rescue experiments that some SoxN neural phenotypes cannot be compensated by Dichaete and that early Dichaete midline functions cannot be fully compensated by SoxN [31].

We uncovered a variety of other binding profile changes indicative of more complex interactions between Drosophila group B proteins. We were surprised to find that loss of binding was the most frequently observed event in both mutant conditions, suggesting a high degree of interdependency between the two factors, a novel aspect of Drosophila SoxB gene biology. At many locations, Dichaete binding appears to be required for the recruitment or the retention of SoxN, and the opposite situation was also observed, though to a lesser extent. It is possible that these observations indicate obligate heterodimerisation at some sites in the genome as occurs with vertebrate group D and E Sox proteins [101]. Alternatively, it may reflect a requirement for interactions with specific cofactors or for the establishment of a suitable chromatin environment by one Sox protein that is necessary for the binding of the other Sox protein. Given the DNA bending properties of the HMG box DNA binding domain [102], it is possible that some of the loss of binding events we observe in mutant embryos are a reflection of Sox-specific chromatin modifications. We also observed increased and de novo binding events in mutant embryos, and in both cases we hypothesise that, in mutant conditions, the remaining Sox protein cannot bind to the vacated locations, but instead occupies nearby open chromatin or increases binding at its normal location to provide sufficient target gene activation.

\section{Conclusions}

Taken together, our studies elucidate the processes coordinated by SoxN during embryogenesis at a genomewide scale and provide evidence for the conservation of SoxB functions in the core regulatory networks underpinning CNS development. We show that, unlike mammalian SoxB1 proteins, SoxN activity is involved in all aspects of neural development, from the initial specification of NBs to their terminal differentiation into mature neural cells. This suggests that Drosophila group B proteins may represent baseline metazoan Sox functions that have been elaborated and diversified as the family expanded in vertebrates. Finally, we provide a detailed genomic perspective on functional redundancy between coexpressed paralogous TFs. We describe genomic regions associated with both redundant and independent functions, uncover evidence for extensive interdependency between the two paralogs and identify key regulatory genes subject to functional compensation, suggesting that redundancy supports the robustness of developmental gene regulatory networks.

\section{Materials and methods}

\section{Fly husbandry and embryo collection}

Fly stocks were obtained from the Cambridge Genetics Department Stock Collection or from the Bloomington Stock Center. Oregon-R was used as wild type. Fly stocks were maintained at $18^{\circ} \mathrm{C}$ or $25^{\circ} \mathrm{C}$ on standard cornmeal medium and dried yeast. Embryo collections were performed at $25^{\circ} \mathrm{C}$ in collection cages on grape agar juice plates supplemented with fresh yeast paste. For all experiments, embryos were collected in Nytex baskets, dechorionated for 5 minutes in $50 \%$ bleach and washed thoroughly with water.

\section{Gene expression experiments}

Embryos from SoxN $^{U 6-35} / C y O$, twi-Gal4 UAS-EGFP X $D f(2$ L)ED647/CyO, twi-Gal4 UAS-EGFP crosses were used to generate gene expression profiles. For stage 10 and older, approximately $200 \mathrm{Sox} \mathrm{N}^{/-}$and $\mathrm{SoxN}^{+/}$embryos per replicate were selected under a fluorescence dissecting microscope on the basis of green fluorescent protein (GFP) expression. For earlier stages of development, a PCR-based method for genotyping single embryos was employed with 12 mutant and control embryos used for each replicate [103]. Microarray hybridization using four biological replicates was performed using our standard protocols [104], with full details provided in the Additional file 13 materials and methods. Scanned images were imported into Dapple [105] for spot finding and quantification, raw data were normalised with the variance stabilization method [106] and statistical analysis of differential expression was carried out using the limma Bioconductor package [107].

\section{Genome-wide binding assays}

Generation of the SoxNDam transgenic line is described in the Additional file 13 materials and methods, and the DichaeteDam line was previously described [49]. Embryos from Dam, SoxNDam, DDam, SoxN $N^{U 6-35} / C y O, D f d-Y F P$; DDam and SoxNDam; $D^{r 72} / T M 6 B$, Dfd-YFP stocks were collected and processed for hybridisation to Nimblegen tiling arrays (GEO platform 15641) using minor modifications to the protocol of Vogel and colleagues [108]. For 
mapping in the wild type we used approximately $2.5 \mathrm{mg}$ dry weight of embryos per replicate; in the case of binding in mutants, approximately 200 YFP-negative embryos at the appropriate stages were selected under a fluorescence dissecting microscope. ChIP followed by hybridisation to Nimblegen tiling arrays was performed with minor modifications to the method described by Sandmann and colleagues [109], as detailed in the Additional file 13 materials and methods. Three biological replicates were performed for all DamID and ChIP experiments. Tiling arrays were quantified using Nimblescan and quantile normalisation was applied to the raw data before using the Ringo Bioconductor package [110] for peak calling at different FDRs. Window score (SGR) and binding interval (BED) files were visualised with the Integrated Genome Browser [111]. The comparative analysis of SoxN and Dichaete binding in wild-type and mutant embryos was performed after all datasets were quantile normalised together. The resulting intensity ratios were used to perform pairwise and three-way comparisons between the datasets with SimBindProfiles [68] as detailed in the Additional file 13 materials and methods.

\section{Other analysis}

The BEDTools suite [112] was used for operations with BED files. Assignment of intervals to genes was performed using a custom script identifying the closest TSS in a $10 \mathrm{~kb}$ window. If no TSSs were found, the interval was assigned to the closest gene boundary in the same $10 \mathrm{~kb}$ window or left otherwise unassigned. GO:BP term enrichment analyses were performed using the BiNGO Cytoscape plugin [113] and corrected for multiple hypothesis testing with the Benjamini-Hochberg method. The HOMER software suite [114] was utilised for both de novo motif discovery and to find enrichment of previously known motifs. Mapping de novo motif matches to the Drosophila genome was done using FIMO at a $P$-value cutoff of 1E-4 [56]. Embryonic binding datasets from the BDTNP $[58,59]$ and modENCODE $[60,61]$ projects were used to identify TF or chromatin feature overlaps using a subsampling-based approach [60,115]. FlyExpress [65] was used for the production of genome-wide expression maps. For network analysis, the whole DroID database [66], with the exception of TF-gene, microRNA-gene and predicted protein-protein interactions was used. The resulted network was imported into Cytoscape [116] and used for further analysis.

\section{Immunohistochemistry and in situ hybridisation}

Embryos from SoxN $N^{U 6-35} / C y O$, twi-Gal4 UAS-EGFP X Df (2 L)ED647/CyO and twi-Gal4 UAS-EGFP or Kr-Gal4/ CyO X UAS-SoxN were collected and processed for antibody staining essentially as described by Patel et al. [117] or for in situ hybridisation as described by Tautz and
Pfeifle [118]. Full details, including the primary antibodies used and their dilutions, are provided in the Additional file 13 materials and methods.

\section{Data access}

All gene expression and ChIP microarray data described in this paper are available from NCBI Gene Expression Omnibus (GEO) in the Superseries accession [GEO: GSE47338].

\section{Additional files}

Additional file 1: Table S1. Expression data, gene lists and Gene Ontology enrichments for genes differentially expressed in SoxN mutants. Additional file 2: Figure S1. Comparison of the different SoxN gene expression and binding datasets.

Additional file 3: Table S2. Genomic coordinates, gene lists and GO:BP enrichments of the SoxN core dataset.

Additional file 4: Figure S2. General features of SoxN binding.

Additional file 5: Figure S3. Comparison of SoxN genome-wide binding with that of other TFs, chromatin-binding proteins and histone modifications.

Additional file 6: Table S3. Genomic coordinates, gene lists, GO:BP enrichments for SoxN direct targets and tables of upregulated, downregulated and variably expressed direct targets.

Additional file 7: Figure S4. Expression of SoxN direct targets in wild-type and SoxN mutant embryos.

Additional file 8: Table S4. Gene lists and GO:BP enrichments for genes bound by SoxN and Sox2 or Sox11.

Additional file 9: Table S5. Genomic coordinates, gene lists and GO:BP enrichments of SoxN and Dichaete binding in wild type and Dichaete and SoxN mutants.

Additional file 10: Figure S5. Differential enrichment of genes targeted by SoxN and Dichaete.

Additional file 11: Figure S6. Differential enrichment of genes associated with the five types of events observed in SoxN and Dichaete mutants.

Additional file 12: Table S6. SoxN and Dichaete binding intervals targeting FlyLight enhancers with reported CNS expression.

Additional file 13: Supplementary Methods and Legends.

\section{Abbreviations}

BDTNP: Berkeley Drosophila Transcription Network Project; BP: biological process; ChIP: chromatin immunoprecipitation; CNS: central nervous system; CRM: cis-regulatory module; DamID: DNA adenine methyltransferase identification; DV: dorsoventral; FDR: false discovery rate; GFP: green fluorescent protein; GO: Gene Ontology; modENCODE: Model Organism Encyclopedia of DNA Elements; NB: neuroblast; NSC: neural stem cell; TF: transcription factor; TSS: transcription start site; UTR: untranslated region; YFP: yellow fluorescent protein.

\section{Competing interests}

The authors declare that they have no competing interests.

\section{Authors' contributions}

EF and SR conceived and designed the experiments; EF performed the experiments; EF, BF and SR analysed the data; EF, BF and SR contributed reagents/material/analysis tools. EF and SR wrote the paper. EF, BF and SR read and approved the final manuscript.

\section{Acknowledgements}

This work was supported by BBSRC and Cambridge Home and European Scholarship Scheme studentships to EF. The funders had no role in study 
design, data collection and analysis, decision to publish, or preparation of the manuscript. We thank Bas van Steensel for providing the pCMycDam vector, Cheng-Yu Lee for the Ase antibody, Ward Odenwald for the Cas antibody, James Skeath for the Dbx, Mid and Spdo antibodies, Chris Doe for the $\mathrm{Hb}$ and Pdm2 antibodies, Mark Biggin for the Hkb and Sna antibodies, Yu Cai for the Insc and Wor antibodies, Adrian Moore for the Kn antibody, Herbert Jackle for the Kr and Run antibodies, Edward Giniger for the Lola antibody, Stephen Crews for the Sim, L'sc and Sc antibodies, Alexander Kuzin for the Nerfin-1 antibody, William Chia for the Nub antibody, Markus Noll for the Poxn antibody, Alex Kolodkin for the Sema-1a antibody and Nicolas Nègre for the SoxND1 and SoxND2 antibodies. We are indebted to Jelena Aleksic, Sarah Bray, Sarah Carl, Alfonso Martinez-Arias and Robert White for comments on the manuscript.

Received: 17 October 2013 Accepted: 30 May 2014

Published: 30 May 2014

\section{References}

1. Peter IS, Davidson $\mathrm{EH}$ : A gene regulatory network controlling the embryonic specification of endoderm. Nature 2011, 474:635-639.

2. Pujato M, MacCarthy T, Fiser A, Bergman A: The underlying molecular and network level mechanisms in the evolution of robustness in gene regulatory networks. PLoS Comp Biol 2013, 9:e1002865

3. Larroux C, Luke GN, Koopman P, Rokhsar DS, Shimeld SM, Degnan BM: Genesis and expansion of metazoan transcription factor gene classes. Mol Biol Evol 2008, 25:980-996.

4. Vavouri T, Semple Jl, Lehner B: Widespread conservation of genetic redundancy during a billion years of eukaryotic evolution. Trends Genet 2008, 24:485-488.

5. Force A, Lynch M, Pickett FB, Amores A, Yan YL, Postlethwait J: Preservation of duplicate genes by complementary, degenerative mutations. Genetics 1999, 151:1531-1545.

6. Wagner A: Distributed robustness versus redundancy as causes of mutational robustness. Bioessays 2005, 27:176-188.

7. Kappen C, Ruddle FH: Evolution of a regulatory gene family: HOM/HOX genes. Curr Opin Genet Dev 1993, 3:931-938.

8. Maconochie M, Nonchev S, Morrison A, Krumlauf R: Paralogous Hox genes: function and regulation. Annu Rev Genet 1996, 30:529-556.

9. Schepers GE, Teasdale RD, Koopman P: Twenty pairs of Sox: extent homology, and nomenclature of the mouse and human sox transcription factor gene families. Dev Cell 2002, 3:167-170.

10. Bowles J, Schepers G, Koopman P: Phylogeny of the SOX family of developmental transcription factors based on sequence and structural indicators. Dev Biol 2000, 227:239-255.

11. Zhong L, Wang D, Gan X, Yang T, He S: Parallel expansions of Sox transcription factor group $B$ predating the diversifications of the arthropods and jawed vertebrates. PLOS One 2011, 6:e16570.

12. Miyagi $\mathrm{S}$, Kato $\mathrm{H}$, Okuda A: Role of SoxB1 transcription factors in development. Cell Mol Life Sci 2009, 66:3675-3684.

13. Penzo-Méndez Al: Critical roles for SoxC transcription factors in development and cancer. Int J Biochem Cell Biol 2010, 42:425-428.

14. Lefebvre V: The SoxD transcription factors - Sox5, Sox6, and Sox13 - are key cell fate modulators. Int J Biochem Cell Biol 2010, 42:429-432.

15. Stolt CC, Wegner M: SoxE function in vertebrate nervous system development. Int J Biochem Cell Biol 2010, 42:437-440.

16. Barrionuevo F, Scherer G: SOX E genes: SOX9 and SOX8 in mammalian testis development. Int J Biochem Cell Biol 2010, 42:433-436.

17. Francois M, Koopman P, Beltrame M: SoxF genes: Key players in the development of the cardio-vascular system. Int J Biochem Cell Biol 2010, 42:445-448.

18. Pontiggia A, Rimini R, Harley VR, Goodfellow PN, Lovell-Badge R, Bianchi ME: Sex-reversing mutations affect the architecture of SRY-DNA complexes. EMBO J 1994, 13:6115-6124.

19. Scaffidi P, Bianchi ME: Spatially precise DNA bending is an essential activity of the sox2 transcription factor. J Biol Chem 2001, 276:47296-47302.

20. Wegner M, Stolt CC: From stem cells to neurons and glia: a Soxist's view of neural development. Trends Neurosci 2005, 28:583-588.

21. Pevny LH, Nicolis SK: Sox2 roles in neural stem cells. Int J Biochem Cell Biol 2010, 42:421-424
22. Uchikawa M, Yoshida M, Iwafuchi-Doi M, Matsuda K, Ishida Y, Takemoto T, Kondoh H: B1 and B2 Sox gene expression during neural plate development in chicken and mouse embryos: universal versus species-dependent features. Dev Growth Differ 2011, 53:761-771.

23. Ferri ALM, Cavallaro M, Braida D, Di Cristofano A, Canta A, Vezzani A Ottolenghi S, Pandolfi PP, Sala M, DeBiasi S, Nicolis SK: Sox2 deficiency causes neurodegeneration and impaired neurogenesis in the adult mouse brain. Development 2004, 131:3805-3819.

24. Nishiguchi S, Wood H, Kondoh H, Lovell-Badge R, Episkopou V: Sox1 directly regulates the gamma-crystallin genes and is essential for lens development in mice. Genes Dev 1998, 12:776-781.

25. Rizzoti K, Brunelli S, Carmignac D, Thomas PQ, Robinson IC, Lovell-Badge $R$ : SOX3 is required during the formation of the hypothalamo-pituitary axis. Nat Genet 2004, 36:247-255.

26. Okuda Y, Ogura E, Kondoh H, Kamachi Y: B1 SOX coordinate cel specification with patterning and morphogenesis in the early zebrafish embryo. PLoS Genet 2010, 6:e1000936.

27. Phochanukul N, Russell S: No backbone but lots of Sox: Invertebrate Sox genes. Int J Biochem Cell Biol 2010, 42:453-464.

28. McKimmie C, Woerfel G, Russell S: Conserved genomic organisation of Group B Sox genes in insects. BMC Genet 2005, 6:26.

29. Wilson MJ, Dearden PK: Evolution of the insect Sox genes. BMC Evol Biol 2008, 8:120

30. Soriano NS, Russell S: The Drosophila SOX-domain protein Dichaete is required for the development of the central nervous system midline. Development 1998, 125:3989-3996.

31. Overton PM: The Role of Sox Genes in the Development of Drosophila melanogaster. In PhD Thesis. University of Cambridge: 2003.

32. Overton PM, Meadows LA, Urban J, Russell S: Evidence for differential and redundant function of the Sox genes Dichaete and SoxN during CNS development in Drosophila. Development 2002, 129:4219-4228.

33. Buescher M, Hing FS, Chia W: Formation of neuroblasts in the embryonic central nervous system of Drosophila melanogaster is controlled by SoxNeuro. Development 2002, 129:4193-4203.

34. Nambu PA, Nambu JR: The Drosophila fish-hook gene encodes a HMG domain protein essential for segmentation and CNS development. Development 1996, 122:3467-3475.

35. Russell SR, Sanchez-Soriano N, Wright CR, Ashburner M: The Dichaete gene of Drosophila melanogaster encodes a SOX-domain protein required for embryonic segmentation. Development 1996, 122:3669-3676.

36. Crémazy F, Berta P, Girard F: SoxNeuro, a new Drosophila Sox gene expressed in the developing central nervous system. Mech Dev 2000, 93:215-219.

37. Sánchez-Soriano N, Russell S: Regulatory mutations of the Drosophila Sox gene Dichaete reveal new functions in embryonic brain and hindgut development. Dev Biol 2000, 220:307-321.

38. Zhao G, Skeath JB: The Sox-domain containing gene Dichaete/fish-hook acts in concert with vnd and ind to regulate cell fate in the Drosophila neuroectoderm. Development 2002, 129:1165-1174.

39. Shen SP, Aleksic J, Russell S: Identifying targets of the Sox domain protein Dichaete in the Drosophila CNS via targeted expression of dominant negative proteins. BMC Dev Biol 2013, 13:1.

40. Graham V, Khudyakov J, Ellis P, Pevny L: SOX2 functions to maintain neura progenitor identity. Neuron 2003, 39:749-765.

41. Sandberg M, Källström M, Muhr J: Sox21 promotes the progression of vertebrate neurogenesis. Nat Neurosci 2005, 8:995-1001.

42. Maurange C, Cheng L, Gould AP: Temporal transcription factors and their targets schedule the end of neural proliferation in Drosophila. Cell 2008 133:891-902.

43. Ma Y, Certel K, Gao Y, Niemitz E, Mosher J, Mukherjee A, Mutsuddi M, Huseinovic N, Crews ST, Johnson WA, Nambu JR: Functional interactions between Drosophila bHLH/PAS, Sox, and POU transcription factors regulate CNS midline expression of the slit gene. J Neurosci 2000, 20:4596-4605

44. Ambrosetti DC, Schöler HR, Dailey L, Basilico C: Modulation of the activity of multiple transcriptional activation domains by the DNA binding domains mediates the synergistic action of Sox 2 and Oct-3 on the fibroblast growth factor-4 enhancer. J Biol Chem 2000, 275:23387-23397.

45. Niwa H, Miyazaki J, Smith AG: Quantitative expression of Oct-3/4 defines differentiation, dedifferentiation or self-renewal of ES cells. Nat Genet 2000 24:372-376. 
46. Masui S, Nakatake Y, Toyooka Y, Shimosato D, Yagi R, Takahashi K, Okochi H, Okuda A, Matoba R, Sharov AA, Ko MSH, Niwa H: Pluripotency governed by Sox2 via regulation of Oct3/4 expression in mouse embryonic stem cells. Nat Cell Biol 2007, 9:625-635.

47. Briscoe J, Novitch BG: Regulatory pathways linking progenitor patterning, cell fates and neurogenesis in the ventral neural tube. Philos Trans $R$ Soc Lond B Biol Sci 2008, 363:57-70.

48. Bergsland M, Ramsköld D, Zaouter C, Klum S, Sandberg R, Muhr J: Sequentially acting Sox transcription factors in neural lineage development. Genes Dev 2011, 25:2453-2464.

49. Aleksic J, Ferrero E, Fischer B, Shen SP, Russell S: The role of Dichaete in transcriptional regulation during Drosophila embryonic development. BMC Genomics 2013, 14:861.

50. Girard F, Joly W, Savare J, Bonneaud N, Ferraz C, Maschat F: Chromatin immunoprecipitation reveals a novel role for the Drosophila SoxNeuro transcription factor in axonal patterning. Dev Biol 2006, 299:530-542

51. Engelen E, Akinci U, Bryne JC, Hou J, Gontan C, Moen M, Szumska D, Kockx C, van ljcken W, Dekkers DHW, Demmers J, Rijkers E-J, Bhattacharya S, Philipsen S, Pevny LH, Grosveld FG, Rottier RJ, Lenhard B, Poot RA: Sox2 cooperates with Chd7 to regulate genes that are mutated in human syndromes. Nat Genet 2011, 43:607-611.

52. Harley VR, Lovell-Badge R, Goodfellow PN: Definition of a consensus DNA binding site for SRY. Nucleic Acids Res 1994, 22:1500-1501.

53. Mertin S, McDowall SG, Harley VR: The DNA-binding specificity of SOX9 and other SOX proteins. Nucleic Acids Res 1999, 27:1359-1364.

54. Noyes MB, Meng X, Wakabayashi A, Sinha S, Brodsky MH, Wolfe SA: A systematic characterization of factors that regulate Drosophila segmentation via a bacterial one-hybrid system. Nucleic Acids Res 2008, 36:2547-2560

55. Aleksic J: The Role of Dichaete in Transcriptional Regulation During Drosophila Embryonic Development. In PhD Thesis. University of Cambridge; 2011.

56. Grant CE, Bailey TL, Noble WS: FIMO: scanning for occurrences of a given motif. Bioinformatics 2011, 27:1017-1018.

57. Noyes MB, Christensen RG, Wakabayashi A, Stormo GD, Brodsky MH, Wolfe SA: Analysis of homeodomain specificities allows the family-wide prediction of preferred recognition sites. Cell 2008, 133:1277-1289.

58. Li X, MacArthur S, Bourgon R, Nix D, Pollard DA, lyer VN, Hechmer A, Simirenko L, Stapleton M, Luengo Hendriks CL, Chu HC, Ogawa N, Inwood W, Sementchenko V, Beaton A, Weiszmann R, Celniker SE, Knowles DW, Gingeras T, Speed TP, Eisen MB, Biggin MD: Transcription factors bind thousands of active and inactive regions in the Drosophila blastoderm. PLoS Biol 2008, 6:e27.

59. MacArthur S, Li X-Y, Li J, Brown JB, Chu HC, Zeng L, Grondona BP, Hechmer A, Simirenko L, Keränen SVE, Knowles DW, Stapleton M, Bickel P, Biggin MD, Eisen MB: Developmental roles of 21 Drosophila transcription factors are determined by quantitative differences in binding to an overlapping set of thousands of genomic regions. Genome Biol 2009, 10:R80.

60. Nègre $N$, Brown CD, Ma L, Bristow CA, Miller SW, Wagner $U$, Kheradpour $P$, Eaton ML, Loriaux P, Sealfon R, Li Z, Ishii H, Spokony RF, Chen J, Hwang L, Cheng C, Auburn RP, Davis MB, Domanus M, Shah PK, Morrison CA, Zieba J, Suchy S, Senderowicz L, Victorsen A, Bild NA, Grundstad AJ, Hanley D, MacAlpine DM, Mannervik M, et al: A cis-regulatory map of the Drosophila genome. Nature 2011, 471:527-531.

61. Kharchenko PV, Alekseyenko AA, Schwartz YB, Minoda A, Riddle NC, Ernst J, Sabo PJ, Larschan E, Gorchakov AA, Gu T, Linder-Basso D, Plachetka A, Shanower G, Tolstorukov MY, Luquette LJ, Xi R, Jung YL, Park RW, Bishop EP Canfield TK, Sandstrom R, Thurman RE, MacAlpine DM, Stamatoyannopoulos JA, Kellis M, Elgin SCR, Kuroda MI, Pirrotta V, Karpen GH, Park PJ: Comprehensive analysis of the chromatin landscape in Drosophila melanogaster. Nature 2011, 471:480-485.

62. Bernstein BE, Mikkelsen TS, Xie X, Kamal M, Huebert DJ, Cuff J, Fry B, Meissner A, Wernig M, Plath K, Jaenisch R, Wagschal A, Feil R, Schreiber SL, Lander ES: A bivalent chromatin structure marks key developmental genes in embryonic stem cells. Cell 2006, 125:315-326.

63. Gallo SM, Gerrard DT, Miner D, Simich M, Des Soye B, Bergman CM, Halfon MS: REDfly v3.0: toward a comprehensive database of transcriptional regulatory elements in Drosophila. Nucleic Acids Res 2011, 39:D118-D123.

64. Manning L, Heckscher ES, Purice MD, Roberts J, Bennett AL, Kroll JR, Pollard JL, Strader ME, Lupton JR, Dyukareva AV, Doan PN, Bauer DM, Wilbur AN, Tanner S, Kelly JJ, Lai S-L, Tran KD, Kohwi M, Laverty TR, Pearson JC, Crews ST, Rubin GM, Doe CQ: A resource for manipulating gene expression and analyzing cis-regulatory modules in the Drosophila CNS. Cell Rep 2012, 2:1002-1013.

65. Kumar S, Konikoff C, Van Emden B, Busick C, Davis KT, Ji S, Wu L-W, Ramos H, Brody T, Panchanathan S, Ye J, Karr TL, Gerold K, McCutchan M, Newfeld SJ: FlyExpress: visual mining of spatiotemporal patterns for genes and publications in Drosophila embryogenesis. Bioinformatics 2011, 27:3319-3320.

66. Murali T, Pacifico S, Yu J, Guest S, Roberts GG, Finley RL: DrolD 2011 a comprehensive, integrated resource for protein, transcription factor, RNA and gene interactions for Drosophila. Nucleic Acids Res 2011, 39:D736-D743.

67. Bardet AF, He Q, Zeitlinger J, Stark A: A computational pipeline for comparative ChIP-seq analyses. Nat Protoc 2012, 7:45-61.

68. SimBindProfiles. [http://www.bioconductor.org/packages/2.14/bioc/html/ SimBindProfiles.html]

69. Ciglar L, Furlong EEM: Conservation and divergence in developmental networks: a view from Drosophila myogenesis. Curr Opin Cell Biol 2009 21:754-760

70. Olson EN: Gene regulatory networks in the evolution and development of the heart. Science 2006, 313:1922-1927.

71. Relaix F, Buckingham M: From insect eye to vertebrate muscle: redeployment of a regulatory network. Genes Dev 1999, 13:3171-3178.

72. Davidson EH, Erwin DH: Gene regulatory networks and the evolution of animal body plans. Science 2006, 311:796-800

73. Van Doren M, Bailey AM, Esnayra J, Ede K, Posakony JW: Negative regulation of proneural gene activity: hairy is a direct transcriptional repressor of achaete. Genes Dev 1994, 8:2729-2742.

74. Zhao G, Wheeler SR, Skeath JB: Genetic control of dorsoventral patterning and neuroblast specification in the Drosophila central nervous system. Int J Dev Biol 2007, 51:107-115.

75. Briscoe J, Pierani A, Jessell TM, Ericson J: A homeodomain protein code specifies progenitor cell identity and neuronal fate in the ventral neural tube. Cell 2000, 101:435-445.

76. Cornell RA, Von Ohlen T: Vnd/nkx, ind/gsh, and msh/msx: conserved regulators of dorsoventral neural patterning? Curr Opin Neurobiol 2000, 10:63-71.

77. Suzuki T, Kaido M, Takayama R, Sato M: A temporal mechanism that produces neuronal diversity in the Drosophila visual center. Dev Biol 2013, 380:12-24.

78. Buescher M, Yeo SL, Udolph G, Zavortink M, Yang X, Tear G, Chia W: Binary sibling neuronal cell fate decisions in the Drosophila embryonic central nervous system are nonstochastic and require inscuteable-mediated asymmetry of ganglion mother cells. Genes Dev 1998, 12:1858-1870.

79. Spana EP, Doe CQ: Numb antagonizes Notch signaling to specify sibling neuron cell fates. Neuron 1996, 17:21-26.

80. Skeath JB, Doe CQ: Sanpodo and Notch act in opposition to Numb to distinguish sibling neuron fates in the Drosophila CNS. Development 1998, 125:1857-1865.

81. O'Connor-Giles KM, Skeath JB: Numb inhibits membrane localization of Sanpodo, a four-pass transmembrane protein, to promote asymmetric divisions in Drosophila. Dev Cell 2003, 5:231-243.

82. Cai $Y$, Chia $W$, Yang $X$ : A family of snail-related zinc finger proteins regulates two distinct and parallel mechanisms that mediate Drosophila neuroblast asymmetric divisions. EMBO J 2001, 20:1704-1714.

83. Ashraf SI, Hu X, Roote J, Ip YT: The mesoderm determinant snail collaborates with related zinc-finger proteins to control Drosophila neurogenesis. EMBO J 1999, 18:6426-6438.

84. Cai $Y$, Yu F, Lin S, Chia W, Yang X: Apical complex genes control mitotic spindle geometry and relative size of daughter cells in Drosophila neuroblast and pl asymmetric divisions. Cell 2003, 112:51-62.

85. Jinushi-Nakao S, Arvind R, Amikura R, Kinameri E, Liu AW, Moore AW: Knot/Collier and cut control different aspects of dendrite cytoskeleton and synergize to define final arbor shape. Neuron 2007, 56:963-978.

86. Parker L, Ellis JE, Nguyen MQ, Arora K: The divergent TGF-beta ligand Dawdle utilizes an activin pathway to influence axon guidance in Drosophila. Development 2006, 133:4981-4991.

87. Serpe M, O'Connor MB: The metalloprotease tolloid-related and its TGF-beta-like substrate Dawdle regulate Drosophila motoneuron axon guidance. Development 2006, 133:4969-4979. 
88. Lacin H, Zhu Y, Wilson BA, Skeath JB: dbx mediates neuronal specification and differentiation through cross-repressive, lineage-specific interactions with eve and hb9. Development 2009, 136:3257-3266.

89. Giniger $E$, Tietje $K$, Jan $L Y$, Jan $Y N$ : Iola encodes a putative transcription factor required for axon growth and guidance in Drosophila. Development 1994, 120:1385-1398.

90. Liu Q-X, Hiramoto M, Ueda H, Gojobori T, Hiromi Y, Hirose S: Midline governs axon pathfinding by coordinating expression of two major guidance systems. Genes Dev 2009, 23:1165-1170.

91. Kuzin A, Brody T, Moore AW, Odenwald WF: Nerfin-1 is required for early axon guidance decisions in the developing Drosophila CNS. Dev Biol 2005 277:347-365.

92. Yu HH, Araj HH, Ralls SA, Kolodkin AL: The transmembrane Semaphorin Sema I is required in Drosophila for embryonic motor and CNS axon guidance. Neuron 1998, 20:207-220.

93. Jones BW, Fetter RD, Tear G, Goodman CS: glial cells missing: a genetic switch that controls glial versus neuronal fate. Cell 1995, 82:1013-1023.

94. Hosoya T, Takizawa K, Nitta K, Hotta Y: glial cells missing: a binary switch between neuronal and glial determination in Drosophila. Cell 1995, 82:1025-1036

95. Kammerer M, Giangrande A: Glide2, a second glial promoting factor in Drosophila melanogaster. EMBO J 2001, 20:4664-4673.

96. De laco R, Soustelle L, Kammerer M, Sorrentino S, Jacques C, Giangrande A: Huckebein-mediated autoregulation of Glide/Gcm triggers glia specification. EMBO J 2006, 25:244-254.

97. Wegner M: SOX after SOX: SOXession regulates neurogenesis. Genes Dev 2011, 25:2423-2428

98. Shen SP: Characterisation of Dichaete Functions and Targets During Drosophila Embryonic Development. In PhD Thesis. University of Cambridge; 2006.

99. Overton PM, Chia W, Buescher M: The Drosophila HMG-domain proteins SoxNeuro and Dichaete direct trichome formation via the activation of shavenbaby and the restriction of Wingless pathway activity. Development 2007, 134:2807-2813.

100. Chao AT, Jones WM, Bejsovec A: The HMG-box transcription factor SoxNeuro acts with Tcf to control Wg/Wnt signaling activity. Development 2007, 134:989-997.

101. Kondoh H, Kamachi Y: SOX-partner code for cell specification: Regulatory target selection and underlying molecular mechanisms. Int J Biochem Cell Biol 2010, 42:391-399.

102. Ferrari S, Harley VR, Pontiggia A, Goodfellow PN, Lovell-Badge R, Bianchi ME: SRY, like HMG1, recognizes sharp angles in DNA. EMBO J 1992, 11:4497-4506

103. Ghanim M, White KP: Genotyping method to screen individual Drosophila embryos prior to RNA extraction. Biotechniques 2006, 41:414. 416, 418.

104. FlyChip. [http://www.flychip.org.uk]

105. Buhler J, Ideker T, Haynor D: Dapple: Improved Techniques for Finding Spots on DNA Microarrays. University of Washington Department of Computer Science \& Engineering Technical Report UW-CSE-2000-08-05; 2000.

106. Huber W, von Heydebreck A, Sültmann H, Poustka A, Vingron M: Variance stabilization applied to microarray data calibration and to the quantification of differential expression. Bioinformatics 2002, 18:S96-S104.

107. Smyth G: Limma: linear models for microarray dat. In Bioinformatics and Computational Biology Solutions using R and Bioconductor. Edited by Gentleman R, Carey VJ, Huber W, RA Irizarry S. Dudoit: Springer, New York; 2005:397-420.

108. Vogel MJ, Peric-Hupkes D, van Steensel B: Detection of in vivo protein-DNA interactions using DamID in mammalian cells. Nat Protoc 2007, 2:1467-1478.

109. Sandmann T, Jakobsen JS, Furlong EEM: ChIP-on-chip protocol for genome-wide analysis of transcription factor binding in Drosophila melanogaster embryos. Nat Protoc 2006, 1:2839-2855.

110. Toedling J, Skylar O, Sklyar O, Krueger T, Fischer JJ, Sperling S, Huber W: Ringo-an R/Bioconductor package for analyzing ChIP-chip readouts. BMC Bioinformatics 2007, 8:221.

111. Nicol JW, Helt GA, Blanchard SG, Raja A, Loraine AE: The Integrated Genome Browser: free software for distribution and exploration of genome-scale datasets. Bioinformatics 2009, 25:2730-2731.

112. Quinlan AR, Hall IM: BEDTools: a flexible suite of utilities for comparing genomic features. Bioinformatics 2010, 26:841-842.

113. Maere S, Heymans K, Kuiper M: BiNGO: a Cytoscape plugin to assess overrepresentation of gene ontology categories in biological networks. Bioinformatics 2005, 21:3448-3449.
114. Heinz S, Benner C, Spann N, Bertolino E, Lin YC, Laslo P, Cheng JX, Murre C, Singh $H$, Glass CK: Simple combinations of lineage-determining transcription factors prime cis-regulatory elements required for macrophage and B cell identities. Mol Cell 2010, 38:576-589.

115. Bickel PJ, Boley N, Brown JB, Huang H, Zhang NR: Subsampling methods for genomic inference. Ann App/ Stat 2010, 4:1660-1697.

116. Smoot ME, Ono K, Ruscheinski J, Wang P-L, Ideker T: Cytoscape 2.8: new features for data integration and network visualization. Bioinformatics 2011, 27:431-432

117. Patel NH, Goldstein LSB, Fyrberg EA: Imaging neuronal subsets and other cell types in whole-mount Drosophila embryos and larvae using antibody probes. In Drosophila melanogaster: Practical Uses in Cell and Molecular Biology. Edited by Goldstein LSB, Fyrberg E. San Diego: Academic Press; 1994:445-487.

118. Tautz D, Pfeifle C: A non-radioactive in situ hybridization method for the localization of specific RNAs in Drosophila embryos reveals translational control of the segmentation gene hunchback. Chromosoma 1989, 98:81-85.

doi:10.1186/gb-2014-15-5-r74

Cite this article as: Ferrero et al:: SoxNeuro orchestrates central nervous system specification and differentiation in Drosophila and is only partially redundant with Dichaete. Genome Biology 2014 15:R74.

\section{Submit your next manuscript to BioMed Central and take full advantage of:}

- Convenient online submission

- Thorough peer review

- No space constraints or color figure charges

- Immediate publication on acceptance

- Inclusion in PubMed, CAS, Scopus and Google Scholar

- Research which is freely available for redistribution

Submit your manuscript at www.biomedcentral.com/submit
C) Biomed Central 